\title{
ORTHOMODULAR LATTICES, FOULIS SEMIGROUPS AND DAGGER KERNEL CATEGORIES
}

\author{
BART JACOBS
}

Institute for Computing and Information Sciences (iCIS), Radboud University Nijmegen, The Netherlands

$U R L$ : www.cs.ru.nl/B.Jacobs

\begin{abstract}
This paper is a sequel to 20 and continues the study of quantum logic via dagger kernel categories. It develops the relation between these categories and both orthomodular lattices and Foulis semigroups. The relation between the latter two notions has been uncovered in the 1960s. The current categorical perspective gives a broader context and reconstructs this relationship between orthomodular lattices and Foulis semigroups as special instances.
\end{abstract}

\section{INTRODUCTION}

Dagger kernel categories have been introduced in 20] as a relatively simple setting in which to study categorical quantum logic. These categories turn out to have orthomodular logic built in, via their posets $\mathrm{KSub}(X)$ of kernel subobjects that can be used to interprete predicates on $X$. The present paper continues the study of dagger kernel categories, especially in relation to orthomodular lattices and Foulis semigroups. The latter two notions have been studied extensively in the context of quantum logic. The main results of this paper are as follows.

(1) A special category OMLatGal is defined with orthomodular lattices as objects and Galois connections between them as morphisms; it is shown that:

- OMLatGal is itself a dagger kernel category - with some additional structure such as dagger biproducts, and an opclassifier;

- for each dagger kernel category $\mathbf{D}$ there is a functor $\mathbf{D} \rightarrow$ OMLatGal preserving the dagger kernel structure; hence OMLatGal contains in a sense all dagger kernel categories.

(2) For each object $X$ in a dagger kernel category, the homset $\mathcal{E} n d o(X)=\mathbf{D}(X, X)$ of endo-maps is a Foulis semigroup.

(3) Every Foulis semigroup $S$ yields a dagger kernel category $\mathcal{K}^{\dagger}(S)$ via the "dagger Karoubi" construction $\mathcal{K}^{\dagger}(-)$.

1998 ACM Subject Classification: F.4.1.

Key words and phrases: quantum logic, orthomodular lattice, Foulis semigroup, categorical logic, dagger kernel category. 
Translations between orthomodular lattices and Foulis semigroups have been described in the 1960s, see e.g. [13, 14, 15, 4, 27]. These translations appear as special instances of the above results:

- given a Foulis semigroup $S$, all the kernel posets $\operatorname{KSub}(s)$ are orthomodular lattices, for each object $s \in \mathcal{K}^{\dagger}(S)$ of the associated dagger kernel category (using point (3) mentioned above). For the unit element $s=1$ this yields the "old" translation from Foulis semigroups to orthomodular lattices;

- given an arbitrary orthomodular lattice $X$, the set of (Galois) endomaps $\mathcal{E}$ do $(X)=$ $\operatorname{OMLatGal}(X, X)$ on $X$ in the dagger kernel category OMLatGal forms a Foulis semigroup - using points (1) and (2). Again this is the "old" translation, from orthomodular lattices to Foulis semigroups.

Since dagger kernel categories are essential in these constructions we see (further) evidence of the relevance of categories in general, and of dagger kernel categories in particular, in the setting of quantum (logical) structures.

The paper is organised as follows. Section 2 first recalls the essentials about dagger kernel categories from [20] and also about the (dagger) Karoubi envelope. It shows that dagger kernel categories are closed under this construction. Section 3 introduces the fundamental category OMLatGal of orthomodular lattices with Galois connections between them, investigates some of its properties, and introduces the functor KSub: D $\rightarrow$ OMLatGal for any dagger kernel category D. Subsequently, Section 4 recalls the definition of Foulis semigroups, shows how they arise as endo-homsets in dagger kernel categories, and proves that their dagger Karoubi envelope yields a dagger kernel category. The paper ends with some final remarks and further questions in Section 5 .

Added in print. After this paper has been accepted for publication, it became clear that the category OMLatGal that plays a central role in this paper was already defined some thirty five years ago, namely by Crown in [8]. There however, the category was not investigated systematically, nor its central position in the context of categorical quantum logic (in terms of dagger kernel categories).

\section{DAgGer KeRnel CATEGORIES}

Since the notion of dagger kernel category is fundamental in this paper we recall the essentials from [20], where this type of category is introduced. Further details can be found there.

A dagger kernel category consists of a category $\mathbf{D}$ with a dagger functor $\dagger: \mathbf{D}^{\text {op }} \rightarrow \mathbf{D}$, a zero object $0 \in \mathbf{D}$, and dagger kernels. The functor $\dagger$ is the identity on objects $X \in \mathbf{D}$ and satisfies $f^{\dagger \dagger}=f$ on morphisms $f$. The zero object 0 yields a zero map, also written as 0 , namely $X \rightarrow 0 \rightarrow Y$ between any two objects $X, Y \in \mathbf{D}$. A dagger kernel of a map $f: X \rightarrow Y$ is a kernel map, written as $k: K \bowtie X$, which is - or can be chosen as - a dagger mono, meaning that $k^{\dagger} \circ k=\mathrm{id}_{K}$. Often we write $\operatorname{ker}(f)$ for the kernel of $f$, and $\operatorname{coker}(f)=\operatorname{ker}\left(f^{\dagger}\right)^{\dagger}$ for its cokernel. The definition $k^{\perp}=\operatorname{ker}\left(k^{\dagger}\right)$ for a kernel $k$ yields an orthocomplement.

We write DKC for the category with dagger kernel categories as objects and functors preserving $\dagger, 0$, ker. 
The main examples of dagger kernel categories are: Rel, the category of sets and relations, its subcategory pInj of sets and partial injections, Hilb, the category of Hilbert spaces and bounded/continuous linear maps between them, and PHilb, the category of projective Hilbert spaces. This paper adds another example, namely OMLatGal.

The main results from [20] about dagger kernel categories are as follows.

(1) Each poset $\operatorname{KSub}(X)$ of kernel subobjects of an object $X$ is an orthomodular lattice; this is the basis of the relevance of dagger kernel categories to quantum logic.

(2) Pullbacks of kernels exist along arbitrary maps $f: X \rightarrow Y$, yielding a pullback (or substitution) functor $f^{-1}: \operatorname{KSub}(Y) \rightarrow \operatorname{KSub}(X)$. Explicitly, as in [16], $f^{-1}(n)=$ $\operatorname{ker}(\operatorname{coker}(n) \circ f)$.

(3) This pullback functor $f^{-1}$ has a left adjoint $\exists_{f}: \operatorname{KSub}(X) \rightarrow \operatorname{KSub}(Y)$, corresponding to image factorisation. These $f^{-1}$ and $\exists_{f}$ only preserve part of the logical structuremeets are preserved by $f^{-1}$ and joins by $\exists_{f}$, via the adjointness - but for instance negations and joins are not preserved by substitution $f^{-1}$, unlike what is standard in categorical logic, see e.g. [26].

Substitution $f^{-1}$ and existential quantification $\exists_{f}$ are inter-expressible, via the equation $f^{-1}(m)^{\perp}=\exists_{f^{\dagger}}\left(m^{\perp}\right)$.

(4) The logical "Sasaki" hook $\supset$ and "and-then" \& connectives - together with the standard adjunction between them [12, 7] - arise via this adjunction $\exists_{f} \dashv f^{-1}$, namely for $m, n, k \in \mathrm{KSub}(X)$ as:

$$
\begin{aligned}
m \supset n & =\mathfrak{E}(m)^{-1}(n) & k \& m & =\exists_{\mathfrak{E}(m)}(k) \\
& =m^{\perp} \vee(m \wedge n) & & =m \wedge\left(m^{\perp} \vee k\right),
\end{aligned}
$$

where $\mathfrak{E}(m)=m \circ m^{\dagger}: X \rightarrow X$ is the effect (see [11]) associated with the kernel $m$.

2.1. Karoubi envelope. Next we recall the essentials of the so-called Karoubi envelope (see [28] or [16, Chapter 2, Exercise B]) construction - and its "dagger" version-involving the free addition of splittings of idempotents to a category. The construction will be used in Section 4 to construct a dagger kernel category out of a Foulis semigroup. It is thus instrumental, and not studied in its own right.

An idempotent in a category is an endomap $s: X \rightarrow X$ satisfying $s \circ s=s$. A splitting of such an idempotent $s$ is a pair of maps $e: X \rightarrow Y$ and $m: Y \rightarrow X$ with $m \circ e=s$ and $e \circ m=\operatorname{id}_{Y}$. Clearly, $m$ is then a mono and $e$ is an epi. Such a splitting, if it exists, is unique up to isomorphism.

For an arbitrary category $\mathbf{C}$ the so-called Karoubi envelope $\mathcal{K}(\mathbf{C})$ has idempotents $s: X \rightarrow X$ in $\mathbf{C}$ as objects. A morphism $(X \stackrel{s}{\rightarrow} X) \stackrel{f}{\longrightarrow}(Y \stackrel{t}{\rightarrow} Y)$ in $\mathcal{K}(\mathbf{C})$ consists of a map $f: X \rightarrow Y$ in $\mathbf{C}$ with $f \circ s=f=t \circ f$. The identity on an object $(X, s) \in \mathcal{K}(\mathbf{C})$ is the map $s$ itself. Composition in $\mathcal{K}(\mathbf{C})$ is as in $\mathbf{C}$. The mapping $X \mapsto\left(X, \operatorname{id}_{X}\right)$ thus yields a full and faithful functor $\mathcal{I}: \mathbf{C} \rightarrow \mathcal{K}(\mathbf{C})$.

The Karoubi envelope $\mathcal{K}(\mathbf{C})$ can be understood as the free completion of $\mathbf{C}$ with splittings of idempotents. Indeed, an idempotent $f:(X, s) \rightarrow(X, s)$ in $\mathcal{K}(\mathbf{C})$ can be split as $f=((X, s) \stackrel{f}{\rightarrow}(X, f) \stackrel{f}{\rightarrow}(X, s))$. If $F: \mathbf{C} \rightarrow \mathbf{D}$ is a functor to a category $\mathbf{D}$ in which endomorphisms split, then there is an up to isomorphism unique functor $\bar{F}: \mathcal{K}(\mathbf{C}) \rightarrow \mathbf{D}$ with $\bar{F} \circ \mathcal{I} \cong F$.

Hayashi [18] (see also 23]) has developed a theory of semi-functors and semi-adjunctions that can be used to capture non-extensional features, without uniqueness of mediating maps, 
like for exponents $\Rightarrow$ [32, 29], products $\prod$ [25], or exponentials! [22]. The Karoubi envelope can be used to turn such "semi" notions into proper (extensional) ones. This also happens in Section 4 .

Now assume $\mathbf{D}$ is a dagger category. An endomap $s: X \rightarrow X$ in $\mathbf{D}$ is called a selfadjoint idempotent if $s^{\dagger}=s=s \circ s$. A splitting of such an $s$ consists, as before, of maps $m, e$ with $m \circ e=s$ and $e \circ m=$ id. In that case $e^{\dagger}, m^{\dagger}$ is also a splitting of $s$, so that we get an isomorphism $\varphi=m^{\dagger} \circ m$ in a commuting diagram:

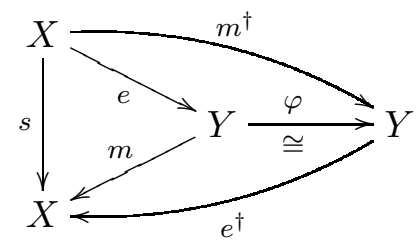

Hence $e^{\dagger}=m$, as subobjects, and $m^{\dagger}=e$ as quotients.

The dagger Karoubi envelope $\mathcal{K}^{\dagger}(\mathbf{D})$ of $\mathbf{D}$ is the full subcategory of $\mathcal{K}(\mathbf{D})$ with selfadjoint idempotents as objects, see also [34]. This is again a dagger category, since if $f:(X, s) \rightarrow(Y, t)$ in $\mathcal{K}^{\dagger}(\mathbf{D})$, then $f^{\dagger}:(Y, t) \rightarrow(X, s)$ because:

$$
s \circ f^{\dagger}=s^{\dagger} \circ f^{\dagger}=(f \circ s)^{\dagger}=f^{\dagger},
$$

and similarly $f^{\dagger} \circ t=f^{\dagger}$. The functor $\mathcal{I}: \mathbf{D} \rightarrow \mathcal{K}(\mathbf{D})$ factors via $\mathcal{K}^{\dagger}(\mathbf{D}) \rightarrow \mathcal{K}(\mathbf{D})$. One can understand $\mathcal{K}^{\dagger}(\mathbf{D})$ as the free completion of $\mathbf{D}$ with splittings of self-adjoint idempotents.

Selinger [34] shows that the dagger Karoubi envelope construction $\mathcal{K}^{\dagger}(-)$ preserves dagger biproducts and dagger compact closedness. Here we extend this with dagger kernels in the next result. It will not be used in the sequel but is included to show that the dagger Karoubi envelope is quite natural in the current setting.

Proposition 2.1. If $\mathbf{D}$ is a dagger kernel category, then so is $\mathcal{K}^{\dagger}(\mathbf{D})$. Moreover, the embedding $\mathcal{I}: \mathbf{D} \rightarrow \mathcal{K}^{\dagger}(\mathbf{D})$ is a map of dagger kernel categories.

Proof For each object $X \in \mathbf{D}$, the zero map $0: X \rightarrow X$ is a zero object in $\mathcal{K}^{\dagger}(\mathbf{D})$, since there is precisely one map $(X, 0) \rightarrow(Y, t)$ in $\mathcal{K}^{\dagger}(\mathbf{D})$, namely the zero map $0: X \rightarrow Y$. As canonical choice we take the zero object $0 \in \mathbf{D}$ with zero map $0=\operatorname{id}_{0}: 0 \rightarrow 0$, which is in the range of $\mathcal{I}: \mathbf{D} \rightarrow \mathcal{K}^{\dagger}(\mathbf{D})$.

For an arbitrary map $f:(X, s) \rightarrow(Y, t)$ in $\mathcal{K}^{\dagger}(\mathbf{D})$, let $k: K \longmapsto X$ be the kernel of $f: X \rightarrow Y$ in D. We obtain a map $s^{\prime}: K \rightarrow K$, as in:

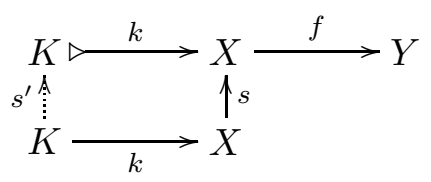


since $f \circ s \circ k=f \circ k=0$. We obtain that $s^{\prime}$ is a self-adjoint idempotent, using that $k$ is a dagger mono (i.e. satisfies $k^{\dagger} \circ k=\mathrm{id}$ ).

$$
\begin{array}{rlrl}
s^{\prime} \circ s^{\prime} & =k^{\dagger} \circ k \circ s^{\prime} \circ s^{\prime} & s^{\prime \dagger} & =s^{\dagger} \circ k^{\dagger} \circ k \\
& =k^{\dagger} \circ s \circ k \circ s^{\prime} & & \left(k \circ s^{\prime}\right)^{\dagger} \circ k \\
& =k^{\dagger} \circ s \circ s \circ k & & (s \circ k)^{\dagger} \circ k \\
& =k^{\dagger} \circ s \circ k & & k^{\dagger} \circ s^{\dagger} \circ k \\
& =k^{\dagger} \circ k \circ s^{\prime} & & k^{\dagger} \circ s \circ k \\
& =s^{\prime} . & & =k^{\dagger} \circ k \circ s^{\prime} \\
& & =s^{\prime} .
\end{array}
$$

This yields a dagger kernel in $\mathcal{K}^{\dagger}(\mathbf{D})$,

$$
\left(K, s^{\prime}\right) \stackrel{s \circ k}{\longrightarrow}(X, s) \stackrel{f}{\longrightarrow}(Y, t)
$$

since:

- $s \circ k$ is a morphism in $\mathcal{K}^{\dagger}(\mathbf{D}): s \circ(s \circ k)=s \circ k$ and $(s \circ k) \circ s^{\prime}=s \circ s \circ k=s \circ k$;

- $s \circ k$ is a dagger mono:

$$
\begin{aligned}
(s \circ k)^{\dagger} \circ(s \circ k) & =\left(k \circ s^{\prime}\right)^{\dagger} \circ\left(k \circ s^{\prime}\right) \\
& =s^{\prime \dagger} \circ k^{\dagger} \circ k \circ s^{\prime} \\
& =s^{\prime} \circ s^{\prime} \\
& =s^{\prime} \\
& =\operatorname{id}_{\left(K, s^{\prime}\right)}
\end{aligned}
$$

- $f \circ(s \circ k)=f \circ k=0$;

- if $g:(Z, r) \rightarrow(X, s)$ satisfies $f \circ g=0$, then there is a map $h: Z \rightarrow K$ in $\mathbf{D}$ with $k \circ h=g$. Then $s^{\prime} \circ h=h$, since $k \circ s^{\prime} \circ h=s \circ k \circ h=s \circ g=g=k \circ h$. Similarly, $h \circ r=h$, since $k \circ h \circ r=g \circ r=g=k \circ h$. Hence $h$ is a morphism $(Z, r) \rightarrow\left(K, s^{\prime}\right)$ in $\mathcal{K}^{\dagger}(\mathbf{D})$ with $(s \circ k) \circ h=s \circ g=g$. It is the unique such mapping with this property since $s \circ k$ is a (dagger) mono.

Example 2.2. In the category Hilb self-adjoint idempotents $s: H \rightarrow H$ are also called projections. They can be written as $s=m \circ m^{\dagger}=\mathfrak{E}(m)$ for a closed subspace $m: M \longmapsto H$, see any textbook on Hilbert spaces (e.g. [10]). Hence they split already in Hilb, and so the dagger Karoubi envelope $\mathcal{K}^{\dagger}$ (Hilb) is isomorphic to Hilb: it does not add anything.

For the category Rel of sets and relations the sitation is different. A self-adjoint idempotent $S: X \rightarrow X$ is a relation $S \subseteq X \times X$ that is both symmetric (since $S^{\dagger}=S$ ) and transitive (since $S \circ S=S$ ), and thus a "partial equivalence relation", commonly abbreviated as PER. The dagger Karoubi envelope $\mathcal{K}^{\dagger}(\mathbf{R e l})$ has such PERs as objects. A morphism $R:(S \subseteq X \times X) \rightarrow(T \subseteq Y \times Y)$ is a relation $R: X \rightarrow Y$ with $R \circ S=R=T \circ R$.

Finally we describe the "effect" operation $\mathfrak{E}(m)=m \circ m^{\dagger}$ as a functor into a dagger Karoubi envelope. For a dagger kernel category $\mathbf{D}$ write $\operatorname{KSub}(\mathbf{D})$ for the category with kernels $M \longmapsto X$ as objects. A morphism $(M \stackrel{m}{\longmapsto} X) \stackrel{f}{\longrightarrow}(N \stackrel{n}{\longmapsto} Y)$ in $\operatorname{KSub}(\mathbf{D})$ is a map $f: X \rightarrow Y$ such that $f \circ m$ factors through $n$. The effect operation can then be described 
as a functor:

$$
\operatorname{KSub}(\mathbf{D}) \stackrel{\mathfrak{E}(-)}{\longrightarrow} \mathcal{K}^{\dagger}(\mathbf{D})
$$

via:

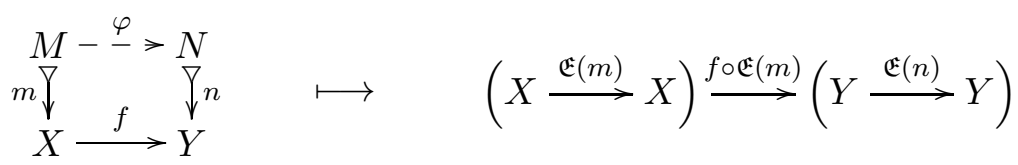

We use that the necessarily unique map $\varphi: M \rightarrow N$ with $n \circ \varphi=f \circ m$ satisfies $\varphi=n^{\dagger} \circ$ $n \circ \varphi=n^{\dagger} \circ f \circ m$. Hence:

$$
\mathfrak{E}(n) \circ f \circ \mathfrak{E}(m)=n \circ n^{\dagger} \circ f \circ m \circ m^{\dagger}=n \circ \varphi \circ m^{\dagger}=f \circ m \circ m^{\dagger}=f \circ \mathfrak{E}(m),
$$

so that $f \circ \mathfrak{E}(m)$ is a morphism $\mathfrak{E}(m) \rightarrow \mathfrak{E}(n)$ in the dagger Karoubi envelope $\mathcal{K}^{\dagger}(\mathbf{D})$. It is not hard to see that this functor is full.

\section{Orthomodular lattices and Dagger kernel CATEgories}

In 20] it was shown how each dagger kernel category gives rise to an indexed collection of orthomodular lattices, given by the posets of the kernel subobjects $\operatorname{KSub}(X)$ of each object $X$. Here we shall give a more systematic description of the situation and see that a suitable category OMLatGal of orthomodular lattices - with Galois connections between them - is itself a dagger kernel category. The mapping $\mathrm{KSub}(-)$ turns out to be functor to this category OMLatGal, providing a form of representation of dagger kernel categories.

We start by recalling the basic notion of orthomodular lattices. They may be understood as a non-distributive generalisation of Boolean algebras. The orthomodularity formulation is due to [24], following [3].

Definition 3.1. A meet semi-lattice $(X, \wedge 1)$ is called an ortholattice if it comes equipped with a function $(-)^{\perp}: X \rightarrow X$ satisfying:

- $x^{\perp \perp}=x$;

- $x \leq y$ implies $y^{\perp} \leq x^{\perp}$;

- $x \wedge x^{\perp}$ is below every element, i.e. is bottom element 0 .

One can thus define a bottom element as $0=1 \wedge 1^{\perp}=1^{\perp}$ and a join as $x \vee y=\left(x^{\perp} \wedge y^{\perp}\right)^{\perp}$, satisfying $x \vee x^{\perp}=1$.

Such an ortholattice is called orthomodular if it satisfies (one of) the three equivalent conditions:

- $x \leq y$ implies $y=x \vee\left(x^{\perp} \wedge y\right)$;

- $x \leq y$ implies $x=y \wedge\left(y^{\perp} \vee x\right)$;

- $x \leq y$ and $x^{\perp} \wedge y=0$ implies $x=y$.

We shall consider two ways of organising orthomodular lattices into a category.

Definition 3.2. The categories OMLat and OMLatGal both have orthomodular lattices as objects.

(1) A morphism $f: X \rightarrow Y$ in OMLat is a function $f: X \rightarrow Y$ between the underlying sets that preserves $\wedge, 1,(-)^{\perp}$-and thus also $\leq, \vee$ and 0 ; 
(2) A morphism $X \rightarrow Y$ in OMLatGal is a pair $f=\left(f_{*}, f^{*}\right)$ of "antitone" functions $f_{*}: X^{\mathrm{op}} \rightarrow Y$ and $f^{*}: Y \rightarrow X^{\mathrm{op}}$ forming a Galois connection (or adjunction $f^{*} \dashv f_{*}$ ): $x \leq f^{*}(y)$ iff $y \leq f_{*}(x)$ for $x \in X$ and $y \in Y$.

The identity morphism on $X$ is the pair $(\perp, \perp)$ given by the self-adjoint map id* $=$ $\mathrm{id}_{*}=(-)^{\perp}: X^{\mathrm{op}} \rightarrow X$. Composition of $X \stackrel{f}{\rightarrow} Y \stackrel{(g}{\rightarrow} Z$ is given by:

$$
(g \circ f)_{*}=g_{*} \circ \perp \circ f_{*} \quad \text { and } \quad(g \circ f)^{*}=f^{*} \circ \perp \circ g^{*} .
$$

The category OMLat is the more obvious one, capturing the (universal) algebraic notion of morphism as structure preserving mapping. However, the category OMLatGal has more interesting structure, as we shall see. It arises by restriction from a familiar construction to obtain a (large) dagger category with involutive categories as objects and adjunctions between them, see [19]. The components $f_{*}: X^{\mathrm{op}} \rightarrow Y$ and $f^{*}: Y \rightarrow X^{\mathrm{op}}$ of a map $f: X \rightarrow Y$ in OMLatGal are not required to preserve any structure, but the Galois connection yields that $f_{*}$ preserves meets, as right adjoint, and thus sends joins in $X$ (meets in $X^{\mathrm{op}}$ ) to meets in $Y$, and dually, $f^{*}$ preserves joins and sends joins in $Y$ to meets in $X$.

The category OMLatGal indeed has a dagger, namely by twisting:

$$
\left(f_{*}, f^{*}\right)^{\dagger}=\left(f^{*}, f_{*}\right) .
$$

A morphism $f: X \rightarrow Y$ in OMLatGal is a dagger mono precisely when it safisfies $f^{*}\left(f_{*}(x)^{\perp}\right)=x^{\perp}$ for all $x \in X$, because $\mathrm{id}^{*}(x)=x^{\perp}=\mathrm{id}_{*}(x)$ and:

$$
\left(f^{\dagger} \circ f\right)^{*}(x)=f^{*}\left(f_{*}(x)^{\perp}\right)=\left(f^{\dagger} \circ f\right)_{*}(x) .
$$

In a Galois connection like $f^{*} \dashv f_{*}$ one map determines the other. This standard result can be useful in proving equalities. For convenience, we make it explicit.

Lemma 3.3. Suppose we have parallel maps $f, g: X \rightarrow Y$ in OMLatGal. In order to prove $f=g$ it suffices to prove either $f_{*}=g_{*}$ or $f^{*}=g^{*}$.

Proof We shall prove that $f_{*}=g_{*}$ suffices to obtain also $f^{*}=g^{*}$. For all $x \in X$ and $y \in Y$,

$$
x \leq f^{*}(y) \Longleftrightarrow y \leq f_{*}(x)=g_{*}(x) \Longleftrightarrow x \leq g^{*}(y) .
$$

Given $y$ this holds for all $x$, and so in particular for $x=f^{*}(y)$ and $x=g^{*}(y)$, which yields $f^{*}(y)=g^{*}(y)$.

Despite this result we sometimes explicitly write out both equations $f_{*}=g_{*}$ and $f^{*}=$ $g^{*}$, in particular when there is a special argument involved.

The following elementary lemma is fundamental.

Lemma 3.4. Let $X$ be an orthomodular lattice, with element $a \in X$.

(1) The (principal) downset $\downarrow a=\{u \in X \mid u \leq a\}$ is again an orthomodular lattice, with order, conjunctions and disjunctions as in $X$, but with its own orthocomplement $\perp_{a}$ given by $u^{\perp_{a}}=a \wedge u^{\perp}$, where $\perp$ is the orthocomplement from $X$.

(2) There is a dagger mono $\downarrow a \longmapsto X$ in OMLatGal, for which we also write a, with

$$
a_{*}(u)=u^{\perp} \quad \text { and } \quad a^{*}(x)=a \wedge x^{\perp} .
$$

Proof For the first point we check, for $u \in \downarrow a$,

$$
u^{\perp_{a} \perp_{a}}=a \wedge\left(a \wedge u^{\perp}\right)^{\perp}=a \wedge\left(a^{\perp} \vee u\right)=u,
$$


B. JACOBS

by orthomodularity, since $u \leq a$. We get a map in OMLatGal because for arbitrary $u \in \downarrow a$ and $x \in X$,

$$
x \leq a_{*}(u)=u^{\perp} \Longleftrightarrow u \leq x^{\perp} \Longleftrightarrow u \leq a \wedge x^{\perp}=a^{*}(x) .
$$

This map $a: \downarrow a \rightarrow X$ is a dagger mono since:

$$
a^{*}\left(a_{*}(u)^{\perp}\right)=a^{*}\left(u^{\perp \perp}\right)=a^{*}(u)=a \wedge u^{\perp}=u^{\perp_{a}} .
$$

We should emphasise that the equation $u^{\perp_{a} \perp_{a}}=u$ only holds for $u \leq a$, and not for arbitrary elements $u$.

Later, in Proposition 3.9, we shall see that these maps $\downarrow a \longmapsto X$ are precisely the kernels in the category OMLatGal. But we first show that this category has kernels in the first place.

To begin, OMLatGal has a zero object $\underline{0}$, namely the one-element orthomodular lattice $\{*\}$. We can write its unique element as $*=0=1$. Let us show that the lattice $\underline{0}$ is indeed a final object in OMLatGal. Let $X$ be an arbitrary orthomodular lattice. The only function $f_{*}: X \rightarrow \underline{0}$ is $f_{*}(x)=1$. It has an obvious left adjoint $f^{*}: \underline{0} \rightarrow L$ defined by $f^{*}(1)=1$ :

$$
\begin{aligned}
& x \leq f^{*}(1) \\
& \hline 1 \leq 1=
\end{aligned} f_{*}(x)
$$

Likewise, the unique morphism $g: \underline{0} \rightarrow Y$ is given by $g_{*}(1)=1$ and $g^{*}(y)=1$. Hence the zero morphism $z: X \rightarrow Y$ is determined by $z_{*}(x)=1$ and $z^{*}(y)=1$.

Theorem 3.5. The category OMLatGal is a dagger kernel category. The (dagger) kernel of a morphism $f: X \rightarrow Y$ is $k: \downarrow k \rightarrow X$, where $k=f^{*}(1) \in X$, as in Lemma 3.4.

Proof The composition $f \circ k$ is the zero map $\downarrow k \rightarrow Y$. First, for $u \in \downarrow f^{*}(1)$,

$$
(f \circ k)_{*}(u)=f_{*}\left(k_{*}(u)^{\perp}\right)=f_{*}\left(u^{\perp \perp}\right)=f_{*}(u)=1,
$$

because $u \leq f^{*}(1)$ in $X$ and so $1 \leq f_{*}(u)$ in $Y$. And for $y \in Y$,

$$
(f \circ k)^{*}(y)=k^{*}\left(f^{*}(y)^{\perp}\right)=f^{*}(y) \wedge f^{*}(1)=f^{*}(y \vee 1)=f^{*}(1)=k=1_{\downarrow k} .
$$

because $f^{*}: Y \rightarrow X^{\text {op }}$ preserves joins as a left adjoint.

Let $g: Z \rightarrow X$, and suppose $g \circ k=0$. We wish to show that there is a unique morphism $h: Z \rightarrow \downarrow k$ with $g=k \circ h$. We have $f_{*} \circ \perp \circ g_{*}=1$ and $g^{*} \circ \perp \circ f^{*}=1$. Hence for $z \in Z$ we have $1 \leq f_{*}\left(g_{*}(z)^{\perp}\right)$, so $g_{*}(z)^{\perp} \leq f^{*}(1)=k$. Define $h_{*}: Z^{\text {op }} \rightarrow \downarrow k$ by $h_{*}(z)=g_{*}(z) \wedge k$, and define $h^{*}: \downarrow k \rightarrow Z^{\text {op }}$ by $h^{*}(u)=g^{*}(u)$. Then $h^{*} \dashv h_{*}$ since for $u \leq k$ and $z \in Z$ :

$$
\begin{aligned}
& \overline{\overline{\overline{u \leq g^{*}(u)}}}=h^{*}(u) \\
& \overline{\overline{u \leq g_{*}(z) \wedge k}}=h_{*}(z)
\end{aligned}
$$

whence $h$ is a well-defined morphism of OMLatGal. It satisfies:

$$
\begin{aligned}
& (k \circ h)_{*}(z)=k_{*}\left(h_{*}(z)^{\perp_{\downarrow k}}\right) \\
& =k_{*}\left(\left(g_{*}(z) \wedge k\right)^{\perp_{\downarrow k}}\right) \\
& =\left(\left(g_{*}(z) \wedge k\right)^{\perp \downarrow k} \wedge k\right)^{\perp} \\
& =\left(\left(g_{*}(z) \wedge k\right)^{\perp} \wedge k \wedge k\right)^{\perp} \\
& =\left(g_{*}(z) \wedge k\right) \vee k^{\perp} \\
& =g_{*}(z) \text {, }
\end{aligned}
$$


where in the last step we use orthomodularity since $k^{\perp}=f^{*}(1)^{\perp} \leq g_{*}(z)$ because $g_{*}(z)^{\perp} \leq$ $f^{*}(1)=k$ which follows from $1 \leq f_{*}\left(g_{*}(z)^{\perp}\right)$. Hence $h$ is a mediating morphism satisfying $k \circ h=g$. It is the unique such morphism, since $k$ is a (dagger) mono.

For convenience we explicitly describe some of the basic structure that results from dagger kernels, see [20], namely cokernels and factorisations, given by dagger kernels and zero-epis. We start with cokernels and zero-epis.

Lemma 3.6. The cokernel of a map $f: X \rightarrow Y$ in OMLatGal is:

$$
\operatorname{coker}(f)=\left(Y \stackrel{c}{\longrightarrow} \downarrow f_{*}(1)\right) \quad \text { with } \quad\left\{\begin{array}{l}
c_{*}(y)=y^{\perp} \wedge f_{*}(1) \\
c^{*}(v)=v^{\perp}
\end{array}\right.
$$

Then:

$$
f \text { is zero-epi } \stackrel{\text { def }}{\Longleftrightarrow} \operatorname{coker}(f)=0 \Longleftrightarrow f_{*}(1)=0 \text {. }
$$

Proof Since:

$$
\operatorname{coker}(f)=\operatorname{ker}\left(f^{\dagger}\right)^{\dagger}=\left(\downarrow\left(f^{\dagger}\right)^{*}(1) \longmapsto Y\right)^{\dagger}=\left(Y \longrightarrow \downarrow f_{*}(1)\right) .
$$

We recall from [20] that each map $f$ in a dagger kernel category has a zero-epi/kernel factorisation $f=i_{f} \circ e_{f}$. In combination with the factorisation of $f^{\dagger}$ it yields a factorisation $f=i_{f} \circ m_{f} \circ\left(i_{f^{\dagger}}\right)^{\dagger}$ as in:

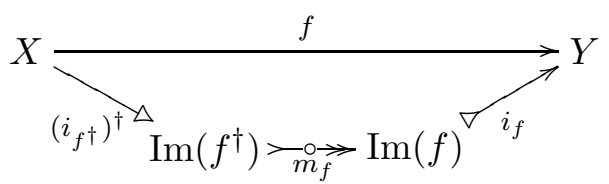

where the map $m_{f}$ is both zero-epic and zero-monic, and where $m_{f} \circ\left(i_{f^{\dagger}}\right)^{\dagger}=e_{f}$, the zero-epic part of $f$.

Lemma 3.7. For a map $f: X \rightarrow Y$ in OMLatGal one has:

$$
\begin{aligned}
& \left(\operatorname{Im}(f)=\downarrow\left(f_{*}(1)^{\perp}\right) \stackrel{i_{f}}{\longrightarrow} Y\right) \quad \text { with } \quad\left\{\begin{array}{l}
\left(i_{f}\right)_{*}(v)=v^{\perp} \\
\left(i_{f}\right)^{*}(y)=y^{\perp} \wedge f_{*}(1)^{\perp}
\end{array}\right. \\
& \left(X \stackrel{e_{f}}{\circ \longrightarrow} \downarrow f_{*}(1)^{\perp}\right) \quad \text { is } \quad\left\{\begin{array}{l}
\left(e_{f}\right)_{*}(x)=f_{*}(x) \wedge f_{*}(1)^{\perp} \\
\left(e_{f}\right)^{*}(v)=f^{*}(v)
\end{array}\right. \\
& \left(\downarrow f^{*}(1)^{\perp} \smile_{0 \rightarrow}^{m_{f}} \downarrow f_{*}(1)^{\perp}\right) \quad \text { is } \quad\left\{\begin{array}{l}
\left(m_{f}\right)_{*}(x)=f_{*}(x) \wedge f_{*}(1)^{\perp} \\
\left(m_{f}\right)^{*}(v)=f^{*}(v) \wedge f^{*}(1)^{\perp} .
\end{array}\right.
\end{aligned}
$$

Proof This is just a matter of unravelling definitions. For instance,

$$
\operatorname{Im}(f)=\operatorname{ker}(\operatorname{coker}(f))=\operatorname{ker}\left(Y \stackrel{c}{\longrightarrow} \downarrow f_{*}(1)\right)=\left(\downarrow f_{*}(1)^{\perp} \longmapsto Y\right) .
$$


since $c^{*}\left(1_{\downarrow f_{*}(1)}\right)=c^{*}\left(f_{*}(1)\right)=f_{*}(1)^{\perp}$. We check that $i_{f} \circ e_{f}=f$, as required.

$$
\begin{aligned}
\left(i_{f} \circ e_{f}\right)_{*}(x) & =\left(i_{f}\right)_{*}\left(\left(e_{f}\right)_{*}(x)^{\perp} f_{*(1)}\right) \\
& =\left(\left(e_{f}\right)_{*}(x)^{\perp} \wedge f_{*}(1)^{\perp}\right)^{\perp} \\
& =\left(f_{*}(x) \wedge f_{*}(1)^{\perp}\right) \vee f_{*}(1) \\
& =f_{*}(x),
\end{aligned}
$$

by orthomodularity, using that $f_{*}(1) \leq f_{*}(x)$, since $x \leq 1$. This map $e_{f}$ is indeed zero-epic by the previous lemma, since:

$$
\left(e_{f}\right)_{*}(1)=f_{*}(1) \wedge f_{*}(1)^{\perp}=0 .
$$

Next we first observe:

$$
f_{*}\left(x \vee f^{*}(1)\right)=f_{*}(x) \wedge f_{*}\left(f^{*}(1)\right)=f_{*}(x) \wedge 1=f_{*}(x),
$$

since there is a "unit" $1 \leq f_{*}\left(f^{*}(1)\right)$. We use this twice, in the marked equations, in:

$$
\begin{aligned}
\left(m_{f} \circ\left(i_{f^{\dagger}}\right)^{\dagger}\right)_{*}(x)= & \left(\left(m_{f}\right)_{*} \circ(-)^{\perp} f^{*(1) \perp} \circ\left(i_{f^{\dagger}}\right)^{*}\right)(x) \\
= & \left(m_{f}\right)_{*}\left(f^{*}(1)^{\perp} \wedge\left(\left(f^{\dagger}\right)_{*}(1)^{\perp} \wedge x^{\perp}\right)^{\perp}\right) \\
= & f_{*}\left(f^{*}(1)^{\perp} \wedge\left(f^{*}(1) \vee x\right)\right) \wedge f_{*}(1)^{\perp} \\
& \stackrel{(*)}{=} f_{*}\left(f^{*}(1) \vee\left(f^{*}(1)^{\perp} \wedge\left(f^{*}(1) \vee x\right)\right) \wedge f_{*}(1)^{\perp}\right. \\
= & f_{*}\left(f^{*}(1) \vee x\right) \wedge f_{*}(1)^{\perp} \\
\stackrel{(*)}{=} & f_{*}(x) \wedge f_{*}(1)^{\perp} \\
= & \left(e_{f}\right)_{*}(x) .
\end{aligned}
$$

The map $m_{f}$ is zero-epic since:

$$
\begin{aligned}
\left(m_{f}\right)_{*}\left(1_{\downarrow f^{*}(1)^{\perp}}\right)=\left(m_{f}\right)_{*}\left(f^{*}(1)^{\perp}\right) & =f_{*}\left(f^{*}(1)^{\perp}\right) \wedge f_{*}(1)^{\perp} \\
& \stackrel{(*)}{=} f_{*}\left(f^{*}(1) \vee f^{*}(1)^{\perp}\right) \wedge f_{*}(1)^{\perp} \\
= & f_{*}(1) \wedge f_{*}(1)^{\perp} \\
= & 0 .
\end{aligned}
$$

Similarly one shows that $m_{f}$ is zero-monic.

For the record, inverse and direct images are described explicitly.

Lemma 3.8. For a map $f: X \rightarrow Y$ in OMLatGal the associated inverse and direct images are:

$$
\begin{array}{ll}
\operatorname{KSub}(Y) \stackrel{f^{-1}}{\longrightarrow} \operatorname{KSub}(X) & \operatorname{KSub}(X) \stackrel{\exists_{f}}{\longrightarrow} \operatorname{KSub}(Y) \\
(\downarrow b \rightarrow Y) \longmapsto\left(\downarrow f^{*}\left(b^{\perp}\right) \rightarrow X\right) & (\downarrow a \rightarrow X) \longmapsto\left(\downarrow\left(f_{*}(a)^{\perp}\right) \rightarrow Y\right)
\end{array}
$$


Proof For $f: X \rightarrow Y$ and $b \in Y$, we have, using the formulation for pullback of kernels from Section 2 (or [20, Lemma 2.4]) and Lemma 3.7 above,

$$
\begin{aligned}
& f^{-1}(\downarrow b \rightarrow Y) \\
& =\operatorname{ker}(\operatorname{coker}(\downarrow b \rightarrow Y) \circ f) \\
& =\operatorname{ker}((Y \rightarrow \downarrow c) \circ f), \quad \text { for } c=b_{*}\left(1_{\downarrow b}\right)=\left(1_{\downarrow b}\right)^{\perp}=b^{\perp} \\
& =\downarrow a \rightarrow X, \quad \text { for } a=\left(c^{\dagger} \circ f\right)^{*}\left(1_{\downarrow b}\right)=f^{*}\left(c_{*}\left(1_{\downarrow c}\right)^{\perp}\right) \\
& =f^{*}\left(c^{\perp \perp}\right)=f^{*}(c)=f^{*}\left(b^{\perp}\right) \\
& =\downarrow f^{*}\left(b^{\perp}\right) \rightarrow X .
\end{aligned}
$$

For $\exists_{f}$ we also use Lemma 3.7 in:

$$
\begin{aligned}
& \exists_{f}(\downarrow a \rightarrow X) \\
& \quad=\operatorname{Im}(f \circ(\downarrow a \rightarrow X)) \\
& \quad=\downarrow b \rightarrow Y, \quad \text { where } b=(f \circ a)_{*}\left(1_{\downarrow a}\right)^{\perp}=f_{*}\left(a_{*}\left(1_{\downarrow a}\right)^{\perp}\right)^{\perp}=f_{*}\left(a^{\perp \perp}\right)^{\perp} \\
& \quad=\downarrow\left(f_{*}(a)^{\perp}\right) \rightarrow Y .
\end{aligned}
$$

As in any dagger kernel category, the kernel posets $\mathrm{KSub}(X)$ of OMLatGal are orthomodular lattices. They turn out to be isomorphic to the underlying object $X \in$ OMLatGal.

Proposition 3.9. Each dagger mono $a: \downarrow a \longmapsto X$ from Lemma 3.4, for $a \in X$, is actually a dagger kernel in OMLatGal. This yields an isomorphism of orthomodular lattices

$$
X \stackrel{\cong}{\longrightarrow} \operatorname{KSub}(X), \quad \text { namely } a \longmapsto(\downarrow a \stackrel{a}{\longrightarrow} X) .
$$

It is natural in the sense that for $f: X \rightarrow Y$ in OMLatGal the following squares commute, by Lemma 3.8 .

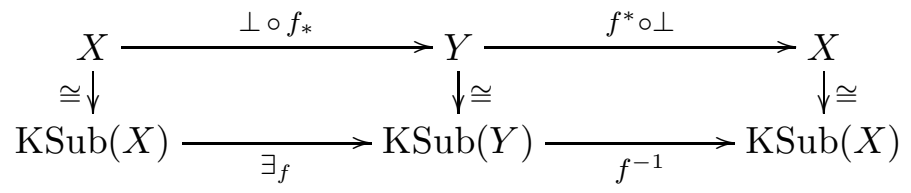

Proof We first check that the map $a: \downarrow a \rightarrow X$ is indeed a kernel, namely of its cokernel $\operatorname{coker}(a): X \rightarrow \downarrow a_{*}(1)$, see Lemma 3.6, where $a_{*}(1)=a_{*}\left(1_{\downarrow}\right)=a_{*}(a)=a^{\perp}$. Thus, $\operatorname{ker}(\operatorname{coker}(a))=\operatorname{coker}(a)^{*}(1)=\operatorname{coker}(a)^{*}\left(1_{\downarrow a^{\perp}}\right)=\operatorname{coker}(a)^{*}\left(a^{\perp}\right)=a^{\perp \perp}=a$.

Theorem 3.5 says that the mapping $X \rightarrow \operatorname{KSub}(X)$ is surjective. Here we shall show that it is an injective homomorphism of orthomodular lattices reflecting the order, so that it is an isomorphism in the category OMLat.

Assume that $a \leq b$ in $X$. We can define $\varphi: \downarrow a \rightarrow \downarrow b$ by $\varphi_{*}(x)=x^{\perp_{b}}=b \wedge x^{\perp}$ and $\varphi^{*}(y)=a \wedge y^{\perp}$, for $x \in \downarrow a$ and $y \in \downarrow b$. Then, clearly, $y \leq \varphi_{*}(x)$ iff $x \leq \varphi^{*}(y)$, so that $\varphi$ is a morphism in OMLatGal. In order to show $a \leq b \operatorname{in~} \operatorname{KSub}(X)$ we prove $b \circ \varphi=a$. First, 
for $x \in \downarrow a$,

$$
\begin{aligned}
(b \circ \varphi)_{*}(x) & =b_{*}\left(\varphi_{*}(x)^{\perp_{b}}\right) \\
& =b_{*}\left(x^{\perp_{b} \perp_{b}}\right) \\
& =b_{*}(x) \quad \text { because } x \in \downarrow a \subseteq \downarrow b \\
& =x^{\perp} \\
& =a_{*}(x) .
\end{aligned}
$$

The map $X \rightarrow \operatorname{KSub}(X)$ not only preserves the order, but also reflects it: if we have an arbitrary map $\psi: \downarrow a \rightarrow \downarrow b$ in OMLatGal with $b \circ \psi=a$, then:

$$
\begin{aligned}
a=a^{\perp \perp}=a_{*}(a)^{\perp} & =(b \circ \psi)_{*}(a)^{\perp} \\
& =b_{*}\left(\psi_{*}(a)^{\perp_{b}}\right)^{\perp} \\
& =\psi_{*}(a)^{\perp_{b} \perp \perp} \\
& =\psi_{*}(a)^{\perp_{b}} \\
& =b \wedge \psi_{*}(a)^{\perp} \leq b .
\end{aligned}
$$

This map $X \rightarrow \operatorname{KSub}(X)$ also preserves $\perp$, since

$$
(\downarrow a \longmapsto \stackrel{a}{\longrightarrow} X)^{\perp}=\operatorname{ker}\left(a^{\dagger}\right)=(\downarrow b \longmapsto b
$$

where, according to Theorem 3.5, $b=\left(a^{\dagger}\right)^{*}\left(1_{\downarrow a}\right)=a_{*}(a)=a^{\perp}$.

It remains to show that the mapping $X \rightarrow \operatorname{KSub}(X)$ preserves finite conjunctions. It is almost immediate that it sends the top element $1 \in X$ to the identity map (top) in $\operatorname{KSub}(X)$. It also preserves finite conjunctions, since the intersection of the kernels $\downarrow a \rightarrow X$ and $\downarrow b \rightarrow X$ is given by $\downarrow(a \wedge b) \rightarrow X$. Since $a \wedge b \leq a, b$ there are appropriate maps $\downarrow(a \wedge b) \rightarrow \downarrow a$ and $\downarrow(a \wedge b) \rightarrow \downarrow b$. Suppose that we have maps $k \rightarrow \downarrow a$ and $k \rightarrow \downarrow b$, where $k: \downarrow f^{*}(1) \rightarrow X$ is a kernel of $f: X \rightarrow Y$. Since, as we have seen, the order is reflected, we get $f^{*}(1) \leq a, b$, and thus $f^{*}(1) \leq a \wedge b$, yielding the required map $\downarrow f^{*}(1) \rightarrow \downarrow(a \wedge b)$.

The adjunction $\exists_{f} \dashv f^{-1}$ that exists in arbitrary dagger kernel categories (see Section 2 or [20, Proposition 4.3]) boils down in our example OMLatGal to the adjunction between $f^{*} \dashv f_{*}$ in the definition of morphisms in OMLatGal, since:

$$
\begin{aligned}
\exists_{f}(\downarrow a \rightarrow X) \leq(\downarrow b \rightarrow Y) & \Longleftrightarrow\left(\downarrow f_{*}(a)^{\perp} \rightarrow Y\right) \leq(\downarrow b \rightarrow Y) \\
& \Longleftrightarrow f_{*}(a)^{\perp} \leq b \\
& \Longleftrightarrow b^{\perp} \leq f_{*}(a) \\
& \Longleftrightarrow a \leq f^{*}\left(b^{\perp}\right) \\
& \Longleftrightarrow(\downarrow a \rightarrow X) \leq\left(\downarrow f^{*}\left(b^{\perp}\right) \rightarrow X\right) \\
& \Longleftrightarrow(\downarrow a \rightarrow X) \leq f^{-1}(\downarrow b \rightarrow X) .
\end{aligned}
$$

Moreover, the Sasaki hook $\supset$ and and-then operators \& defined categorically in $[20$, Proposition 6.1], see Section 2, amount in OMLatGal to their usual definitions in the theory of orthomodular lattices, see e.g. [12, 27]. This will be illustrated next. We use the "effect" $\mathfrak{E}(\downarrow a \rightarrow X)=a \circ a^{\dagger}: X \rightarrow X$ associated with a kernel in:

$$
(\downarrow a \rightarrow X) \supset(\downarrow b \rightarrow X) \stackrel{\text { def }}{=} \mathfrak{E}(\downarrow a \rightarrow X)^{-1}(\downarrow b \rightarrow X)=(\downarrow c \rightarrow X),
$$


where, according to the description of inverse image $(-)^{-1}$ in the previous lemma,

$$
c=\left(a \circ a^{\dagger}\right)^{*}\left(b^{\perp}\right)=a_{*}\left(a^{*}\left(b^{\perp}\right)^{\perp_{a}}\right)=\left(a \wedge(a \wedge b)^{\perp}\right)^{\perp}=a^{\perp} \vee(a \wedge b)=a \supset b .
$$

Similarly for and-then $\&$ :

$$
(\downarrow a \rightarrow X) \&(\downarrow b \rightarrow X) \stackrel{\text { def }}{=} \exists_{\mathfrak{E}(\downarrow b \rightarrow X)}(\downarrow a \rightarrow X)=(\downarrow c \rightarrow X),
$$

where the description of direct image from Lemma 3.8 yields:

$$
c=\left(b \circ b^{\dagger}\right)_{*}(a)^{\perp}=b_{*}\left(b^{*}(a)^{\perp}\right)^{\perp}=\left(b \wedge\left(b \wedge a^{\perp}\right)^{\perp}\right)^{\perp \perp}=b \wedge\left(b^{\perp} \vee a\right)=a \& b .
$$

These $\&$ and $\supset$ are, by construction, related via an adjunction (see also [12, 17).

Also one can define a weakest precondition modality $[f]$ from dynamic logic in this setting: for $f: X \rightarrow Y$ and $y \in Y$, put:

$$
[f](y) \stackrel{\text { def }}{=} f^{*}\left(y^{\perp}\right) .
$$

for " $y$ holds after $f$ ". This operation $[f](-)$ preserves conjunctions, as usual. An element $a \in X$ yields a test operation $a ?=\mathfrak{E}(a)=a \circ a^{\dagger}$. Then one can recover the Sasaki hook $a \supset b$ via this modality as $[a ?] b$, and hence complement $a^{\perp}$ as $[a$ ?]0, see also e.g. [2].

There is another isomorphism of interest in this setting.

Lemma 3.10. Let $2=\{0,1\}$ be the 2-element Boolean algebra, considered as an orthomodular lattice $2 \in$ OMLatGal. For each orthomodular lattice $X$, there is an isomorphism (of sets):

$$
X \stackrel{\cong}{\longrightarrow} \text { OMLatGal }(2, X)
$$

which maps $a \in X$ to $\bar{a}: 2 \rightarrow X$ given by:

$$
\bar{a}_{*}(w)=\left\{\begin{array}{ll}
1 & \text { if } w=0 \\
a^{\perp} & \text { if } w=1
\end{array} \quad \bar{a}^{*}(x)= \begin{cases}1 & \text { if } x \leq a^{\perp} \\
0 & \text { otherwise. }\end{cases}\right.
$$

This isomorphism is natural: for $f: X \rightarrow Y$ one has:

$$
\begin{array}{ccc}
X & \perp \circ f_{*} & \\
\cong \downarrow & & \downarrow \\
\text { OMLatGal }(2, X) \longrightarrow & f \circ- & \text { OMLatGal }(2, Y)
\end{array}
$$

Proof The thing to note is that for a map $g: 2 \rightarrow X$ in OMLatGal we have $g_{*}(0)=1$ because $g_{*}: 2^{\text {op }} \rightarrow X$ is a right adjoint. Hence we can only choose $g_{*}(1) \in X$. Once this is chosen, the left adjoint $g^{*}: X \rightarrow 2^{\text {op }}$ is completely determined, namely as $1 \leq g^{*}(x)$ iff $x \leq g_{*}(1)$.

As to naturality, it suffices to show:

$$
\begin{aligned}
(f \circ \bar{a})_{*}(1) & =f_{*}\left(\bar{a}_{*}(1)^{\perp}\right) \\
& =f_{*}\left(a^{\perp \perp}\right) \\
& =f_{*}(a)^{\perp \perp} \\
& =\overline{f *}_{*}(a)^{\perp} \\
& =11) \\
\left(\perp \circ f_{*}\right)(a)_{*} & (1) .
\end{aligned}
$$


By combining the previous two results we obtain a way to classify (kernel) subobjects, as in a topos [30], but with naturality working in the opposite direction. In [20] a similar structure was found in the category Rel of sets and relations, and also in the dagger kernel category associated with a Boolean algebra.

Corollary 3.11. The 2-element lattice $2 \in$ OMLatGal is an "opclassifier": there is a "characteristic" isomorphism:

$$
\operatorname{KSub}(X) \underset{\text { char }}{\cong} \text { OMLatGal }(2, X) .
$$

which is natural: char $\circ \exists_{f}=f \circ$ char.

We conclude our investigation of the category OMLatGal with the following observation.

Proposition 3.12. The category OMLatGal has (finite) dagger biproducts $\oplus$. Explicitly, $X_{1} \oplus X_{2}$ is the Cartesian product of (underlying sets of) orthomodular lattices, with coprojection $\kappa_{1}: X_{1} \rightarrow X_{1} \oplus X_{2}$ defined by $\left(\kappa_{1}\right)_{*}(x)=\left(x^{\perp}, 1\right)$ and $\left(\kappa_{1}\right)^{*}(x, y)=x^{\perp}$. The dual product structure is given by $\pi_{i}=\left(\kappa_{i}\right)^{\dagger}$.

Proof Let us first verify that $\kappa_{1}$ is a well-defined morphism of OMLatGal, i.e. that $\left(\kappa_{1}\right)^{*} \dashv\left(\kappa_{1}\right)_{*}:$

Also, $\kappa_{1}$ is a dagger mono since:

$$
\begin{gathered}
\frac{z \leq x^{\perp}}{\overline{\overline{x \leq z^{\perp}}}}=\left(\kappa_{1}\right)^{*}(x, y) \\
\overline{\overline{(x, y) \leq\left(z^{\perp}, 1\right)}}=\left(\kappa_{1}\right)_{*}(z)
\end{gathered}
$$

$$
\left(\kappa_{1}\right)^{*}\left(\left(\kappa_{1}\right)_{*}(x)^{\perp}\right)=\left(\kappa_{1}\right)^{*}\left(\left(x^{\perp}, 1\right)^{\perp}\right)=\left(\kappa_{1}\right)^{*}(x, 0)=x^{\perp} .
$$

Likewise, there is a dagger mono $\kappa_{2}: X_{2} \rightarrow X_{1} \oplus X_{2}$. For $i \neq j$, one finds that $\left(\kappa_{j}\right)^{\dagger} \circ \kappa_{i}$ is the zero morphism.

In order to show that $X_{1} \oplus X_{2}$ is indeed a coproduct, suppose that morphisms $f_{i}: X_{i} \rightarrow$ $Y$ are given. We then define the cotuple $\left[f_{1}, f_{2}\right]: X_{1} \oplus X_{2} \rightarrow Y$ by $\left[f_{1}, f_{2}\right]_{*}\left(x_{1}, x_{2}\right)=$ $\left(f_{1}\right)_{*}\left(x_{1}\right) \wedge\left(f_{2}\right)_{*}\left(x_{2}\right)$ and $\left[f_{1}, f_{2}\right]^{*}(y)=\left(f_{1}^{*}(y), f_{2}^{*}(y)\right)$. Clearly, $\left[f_{1}, f_{2}\right]^{*} \dashv\left[f_{1}, f_{2}\right]_{*}$, and:

$$
\begin{aligned}
\left(\left[f_{1}, f_{2}\right] \circ \kappa_{1}\right)_{*}(x) & =\left[f_{1}, f_{2}\right]_{*}\left(\left(\kappa_{1}\right)_{*}(x)^{\perp}\right) \\
& =\left[f_{1}, f_{2}\right]_{*}\left(\left(x^{\perp}, 1\right)^{\perp}\right) \\
& =\left(f_{1}\right)_{*}(x) \wedge\left(f_{2}\right)_{*}(0) \\
& =\left(f_{1}\right)_{*}(x) \wedge 1 \\
& =\left(f_{1}\right)_{*}(x) .
\end{aligned}
$$


so that $\left[f_{1}, f_{2}\right] \circ \kappa_{1}=f_{1}$. Likewise, $\left[f_{1}, f_{2}\right] \circ \kappa_{2}=f_{2}$. Moreover, if $g: X_{1} \oplus X_{2} \rightarrow Y$ also satisfies $g \circ \kappa_{i}=f_{i}$, then:

$$
\begin{aligned}
{\left[f_{1}, f_{2}\right]_{*}\left(x_{1}, x_{2}\right) } & =\left(f_{1}\right)_{*}\left(x_{1}\right) \wedge\left(f_{2}\right)_{*}\left(x_{2}\right) \\
& =g_{*}\left(\left(\kappa_{1}\right)_{*}\left(x_{1}\right)^{\perp}\right) \wedge g_{*}\left(\left(\kappa_{2}\right)_{*}\left(x_{2}\right)^{\perp}\right) \\
& =g_{*}\left(\left(x_{1}^{\perp}, 1\right)^{\perp}\right) \wedge g_{*}\left(\left(1, x_{2}^{\perp}\right)^{\perp}\right) \\
& =g_{*}\left(x_{1}, 0\right) \wedge g_{*}\left(0, x_{2}\right) \\
& =g_{*}\left(\left(x_{1}, 0\right) \vee\left(0, x_{2}\right)\right) \\
& =g_{*}\left(x_{1}, x_{2}\right) .
\end{aligned}
$$

3.1. From dagger kernel categories to orthomodular lattices. The aim in this subsection is to show that for an arbitrary dagger kernel $\mathbf{D}$ the kernel subobject functor $\mathrm{KSub}(-)$ is a functor $\mathbf{D} \rightarrow$ OMLatGal. On a morphism $f: X \rightarrow Y$ of $\mathbf{D}$, define $\operatorname{KSub}(f): \operatorname{KSub}(X) \rightarrow \operatorname{KSub}(Y)$ by:

$$
\operatorname{KSub}(f)_{*}(m)=\left(\exists_{f}(m)\right)^{\perp} \quad \operatorname{KSub}(f)^{*}(n)=f^{-1}\left(n^{\perp}\right) .
$$

Then indeed $\operatorname{KSub}(f)^{*} \dashv \operatorname{KSub}(f)_{*}$, via $\exists_{f} \dashv f^{-1}$,

$$
\begin{aligned}
& \frac{n \leq\left(\exists_{f}(m)\right)^{\perp}}{\overline{\exists_{f}(m) \leq n^{\perp}}}=\operatorname{KSub}(f)_{*}(m) \\
& \overline{\overline{m \leq f^{-1}\left(n^{\perp}\right)}}=\operatorname{KSub}(f)^{*}(n)
\end{aligned}
$$

The functor $\mathrm{KSub}(-)$ preserves the relevant structure. This requires the following auxiliary result.

Lemma 3.13. In a dagger kernel category, for any kernel $k: K \longmapsto X$ in $\operatorname{KSub}(X)$, there is an order isomorphism $\mathrm{KSub}(K) \cong \downarrow k \subseteq \mathrm{KSub}(X)$.

Proof The direction $\operatorname{KSub}(K) \rightarrow \downarrow k$ of the desired bijection is given by $m \mapsto k \circ m$. This is well-defined since kernels are closed under composition. The other direction $\downarrow k \rightarrow \operatorname{KSub}(K)$ is $n \mapsto \varphi=k^{\dagger} \circ n$, where $n=k \circ \varphi$. One easily checks that these maps are each other's inverse, and preserve the order.

Theorem 3.14. Let $\mathbf{D}$ be a dagger kernel category. The functor KSub: D $\rightarrow$ OMLatGal is a map of dagger kernel categories.

Proof Preservation of daggers follows because $f^{-1}$ and $\exists_{f}$ are inter-expressible, see Section 2 and [20, Proposition 4.3]:

$$
\operatorname{KSub}\left(f^{\dagger}\right)_{*}(n)=\left(\exists_{f^{\dagger}}(n)\right)^{\perp}=f^{-1}\left(n^{\perp}\right)=\operatorname{KSub}(f)^{*}(n)=\left(\operatorname{KSub}(f)^{\dagger}\right)_{*}(n) .
$$

Preservation of the zero object is easy: $\operatorname{KSub}(0)=\{0\}=\underline{0}$. Next, let $k: K \rightarrow X$ be the kernel of a morphism $f: X \rightarrow Y$ in $\mathbf{D}$. We recall from [20, Corollary 2.5 (ii)] that this kernel $k$ can be described as inverse image $k=f^{-1}(0)=f^{-1}\left(1^{\perp}\right)=\operatorname{KSub}(f)^{*}(1)$. Hence by Lemmas 3.13 and 3.4, we have the isomorphism on the left in:

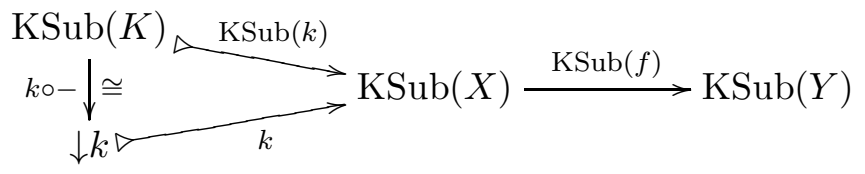


It yields a commuting triangle since for $n \in \operatorname{KSub}(K)$,

$$
\operatorname{KSub}(k)_{*}(n)=\exists_{k}(n)^{\perp}=(k \circ n)^{\perp}=k_{*}(k \circ n) .
$$

Similarly for $m \in \mathrm{KSub}(X)$,

$$
k \circ \operatorname{KSub}(k)^{*}(m)=k \circ k^{-1}\left(m^{\perp}\right)=k \wedge m^{\perp}=k^{*}(m) .
$$

At this stage we conclude that these KSub functors yield a well-behaved translation of a dagger kernel category into a collection of orthomodular lattices, indexed by the objects of the category. For the special case $\mathbf{D}=$ OMLatGal, the functor KSub: D $\rightarrow$ OMLatGal is the identity, up to isomorphism, by Proposition 3.9. A translation in the other direction, from orthomodular lattices to dagger kernel categories will be postponed until after the next section, after we have seen the translation from Foulis semigroups to orthomodular lattices.

Remark 3.15. As pointed out by John Harding, the functor KSub: D $\rightarrow$ OMLatGal need not preserve biproducts that exist in $\mathbf{D}$. For instance if we take $\mathbf{D}$ to be the category of Hilbert spaces over $\mathbb{R}$, then $\operatorname{KSub}(\mathbb{R})$ is a two-element set, containing $\{0\}$ and $\mathbb{R}$ itself. However, $\operatorname{KSub}(\mathbb{R} \oplus \mathbb{R})=\operatorname{KSub}\left(\mathbb{R}^{2}\right)$ is much bigger than $\operatorname{KSub}(\mathbb{R}) \times \operatorname{KSub}(\mathbb{R})$, since every line through the origin forms a closed subspace of $\mathbb{R}^{2}$.

In the remainder of this section we shall briefly consider two special subcategories of OMLatGal, namely with Boolean and with complete orthomodular lattices.

3.2. The Boolean case. Let BoolGal $\hookrightarrow$ OMLatGal be the full subcategory of Boolean algebras with (antitone) Galois connections between them. We recall that a Boolean algebra can be described as an orthomodular lattice that is distributive.

The main (and only) result of this subsection is simple.

Proposition 3.16. The category BoolGal inherits dagger kernels and biproducts from OMLatGal. Moreover, as a dagger kernel category it is Boolean.

Proof An arbitrary map $f: X \rightarrow Y$ in BoolGal has a kernel $\downarrow f^{*}(1) \rightarrow X$ as in Theorem 3.5 for orthomodular lattices because the downset $\downarrow f^{*}(1)$ is a Boolean algebra. Similarly, the biproducts from Proposition 3.12 also exist in BoolGal because $X_{1} \oplus X_{2}$ is a Boolean algebra if $X_{1}$ and $X_{2}$ are Boolean algebras.

For each $X \in \mathbf{B o o l G a l}$ one has $\operatorname{KSub}(X) \cong X$ so that $\operatorname{KSub}(X)$ is a Boolean algebra. Hence BoolGal is a Boolean dagger kernel category by [20, Theorem 6.2].

Boolean algebras thus give rise to (Boolean) dagger kernel categories on two different levels: the "large" category BoolGal of all Boolean algebras is a dagger kernel category, but also each individual Boolean algebra can be turned into a "small" dagger kernel category, see [20, Proposition 3.5].

3.3. Complete orthomodular lattices. We shall write OMSupGal $\hookrightarrow$ OMLatGal for the full subcategory of orthomodular lattices that are complete, i.e. that have joins $\bigvee U$ (and thus also meets $\wedge U$ ) of all subsets $U$ (and not just the finite ones). Notice that the functor KSub: D $\rightarrow$ OMLatGal from Theorem 3.14 is actually a functor KSub: D $\rightarrow$ OMSupGal for $\mathbf{D}=$ Rel, PInj, Hilb. 
A morphism $f: X \rightarrow Y$ in OMSupGal is completely determined by either $f_{*}: X^{\mathrm{op}} \rightarrow$ $Y$ preserving all meets, or by $f^{*}: Y \rightarrow X^{\text {op }}$ preserving all joins. This forms the basis for the next result.

Proposition 3.17. The forgetful functor $U$ : OMSupGal $\rightarrow$ Sets given by $X \mapsto X$ on objects and $f \mapsto f_{*} \circ \perp$ on morphisms has a left adjoint $F$ given by $F(A)=\mathcal{P} A$, with $F(g)_{*}(U \subseteq A)=\neg \coprod_{g}(U)=\neg\{g(a) \mid a \in U\}$ and $F(g)^{*}(V \subseteq B)=g^{-1}(\neg V)=\{a \mid g(a) \notin$ $V\}$, for $g: A \rightarrow B$ in Sets.

Proof For $A \in$ Sets and $X \in \mathbf{O M S u p G a l ~ t h e r e ~ i s ~ a ~ b i j e c t i v e ~ c o r r e s p o n d e n c e : ~}$

$$
\stackrel{\mathcal{P} A \stackrel{f}{\longrightarrow} X}{\stackrel{g}{\longrightarrow} X} \text { in Sets }
$$

given by $\bar{f}(a)=f_{*}(\{a\})^{\perp}$ and $\bar{g}_{*}(U)=\bigwedge_{a \in U} g(a)^{\perp}$ with $\bar{g}^{*}(x)=\left\{a \in A \mid g(a) \leq x^{\perp}\right\}$. Then:

$$
\begin{aligned}
x \leq \bar{g}_{*}(U)=\bigwedge_{a \in U} g(a)^{\perp} & \Longleftrightarrow \forall_{a \in U} \cdot x \leq g(a)^{\perp} \\
& \Longleftrightarrow \forall_{a \in U} \cdot g(a) \leq x^{\perp} \\
& \Longleftrightarrow U \subseteq\left\{a \in A \mid g(a) \leq x^{\perp}\right\}=\bar{g}^{*}(x) .
\end{aligned}
$$

Further,

$$
\begin{aligned}
\overline{\bar{g}}(a) & =\bar{g}_{*}(\{a\})^{\perp}=\left(\bigwedge_{b \in\{a\}} g(b)^{\perp}\right)^{\perp}=g(a)^{\perp \perp}=g(a) . \\
\overline{\bar{f}}_{*}(U) & =\bigwedge_{a \in U} \bar{f}(a)^{\perp}=\bigwedge_{a \in U} f_{*}(\{a\})^{\perp \perp}=f_{*}\left(\bigcup_{a \in U}\{a\}\right)=f_{*}(U) \\
\overline{\bar{f}}^{*}(x) & =\left\{a \mid \bar{f}(a) \leq x^{\perp}\right\}=\left\{a \mid f_{*}(\{a\})^{\perp} \leq x^{\perp}\right\}=\left\{a \mid x \leq f_{*}(\{a\})\right\} \\
& =\left\{a \mid\{a\} \subseteq f^{*}(x)\right\}=f^{*}(x) .
\end{aligned}
$$

The left adjoint $F$ of this adjunction between OMSupGal and Sets factors via the graph functor $\mathcal{G}:$ Sets $\rightarrow$ Rel, as in:

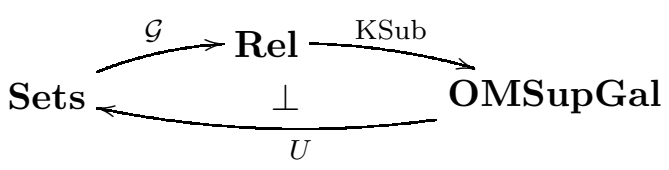

It is not hard to see that the kernels from Theorem 3.5 and biproducts $\oplus$ from Proposition 3.12 also exist in OMSupGal. For instance, the join of a subset $U \subseteq X \oplus Y$ is given as pair of joins:

$$
\bigvee U=\left(\bigvee\left\{x \mid \exists_{y} \cdot(x, y) \in U\right\}, \bigvee\left\{y \mid \exists_{x} .(x, y) \in U\right\}\right)
$$

Hence OMSupGal is also a dagger kernel category with dagger biproducts.

\section{Foulis Semigroups And DAgGer Kernel CATEGories}

In this section we shall relate dagger kernel categories and Foulis semigroups. Postponing the formal definition, we first illustrate that these Foulis semigroups arise quite naturally in the context of kernel dagger categories.

In every category $\mathbf{D}$ the homset $\mathcal{E} n d o(X)=\mathbf{D}(X, X)$ of endomaps $f: X \rightarrow X$ is a monoid (or semigroup with unit), with obvious composition operation $\circ$ and identity map $\operatorname{id}_{X}$ as unit element. If $\mathbf{D}$ is a dagger category, there is automatically an involution $(-)^{\dagger}$ on 
this monoid. If it is moreover a dagger kernel category, every endomap $s \in \mathcal{E} n d o(X)$ yields a self-adjoint idempotent, namely the effect of its kernel:

$$
[s] \stackrel{\text { def }}{=} \mathfrak{E}(\operatorname{ker}(s))=\operatorname{ker}(s) \circ \operatorname{ker}(s)^{\dagger}: X \longrightarrow X
$$

with the special property that for $t \in \mathcal{E} n d o(X)$,

$$
s \circ t=0 \Longleftrightarrow \exists_{r \in \mathcal{E} n d o(X)} \cdot t=[s] \circ r .
$$

Indeed, if $t=[s] \circ r$, then:

$$
s \circ t=s \circ \operatorname{ker}(s) \circ \operatorname{ker}(s)^{\dagger} \circ r=0 \circ \operatorname{ker}(s)^{\dagger} \circ r=0 .
$$

Conversely, if $s \circ t=0$, then there is a map $f$ in $\mathbf{D}$ with $\operatorname{ker}(s) \circ f=t$. Hence $t$ satisfies:

$$
[s] \circ t=\operatorname{ker}(s) \circ \operatorname{ker}(s)^{\dagger} \circ \operatorname{ker}(s) \circ f=\operatorname{ker}(s) \circ f=t .
$$

In the 1960s this structure of an involutive monoid $\langle\mathcal{E} n d o(X), \circ, \mathrm{id}, \dagger\rangle$ with operation $[-]: \mathcal{E}$ do $(X) \rightarrow \mathcal{E}$ ndo $(X)$ was introduced by Foulis [13, 14, 15] and has since been studied under the name 'Baer *-semigroup', and later as 'Foulis semigroup', see [27, Chapter 5, $\S \S 18]$ for a brief overview.

Definition 4.1. A Foulis semigroup consists of a monoid (semigroup with unit) $(S, \cdot, 1)$ together with two endomaps $(-)^{\dagger}: S \rightarrow S$ and [-]:S $\rightarrow S$ satisfying:

(1) $1^{\dagger}=1$ and $(s \cdot t)^{\dagger}=t^{\dagger} \cdot s^{\dagger}$ and $s^{\dagger \dagger}=s$, making $S$ an involutive monoid;

(2) $[s]$ is a self-adjoint idempotent, i.e. satisfies $[s] \cdot[s]=[s]=[s]^{\dagger}$;

(3) $0 \stackrel{\text { def }}{=}[1]$ is a zero element: $0 \cdot s=0=s \cdot 0$;

(4) $s \cdot x=0$ iff $\exists_{y} \cdot x=[s] \cdot y$.

Or, equivalently (see [27, Chapter 5, §§18, Lemma 1]),

(4)' $[0]=1$ and $s \cdot[s]=0$ and $t=\left[\left[t^{\dagger} \cdot s^{\dagger}\right] \cdot s\right] \cdot t$.

We form a category Fsg of such Foulis semigroups with monoid homomorphisms that commute with $\dagger$ and $[-]$ as morphisms.

The constructions before this definition show that for each object $X \in \mathbf{D}$ of a (locally small) dagger kernel category $\mathbf{D}$, the homset $\mathcal{E} n d o(X)=\mathbf{D}(X, X)$ of endomaps on $X$ is a Foulis semigroup. Functoriality of this construction is problematic: for an arbitrary map $f: X \rightarrow Y$ in $\mathbf{D}$ there is a mapping $\mathcal{E} n d o(X) \rightarrow \mathcal{E} n d o(Y)$, namely $s \mapsto f \circ s \circ f^{\dagger}: Y \rightarrow X \rightarrow$ $X \rightarrow Y$, but it does not preserve the structure of Foulis semigroups, and thus only gives rise to presheaf.

Proposition 4.2. For a dagger kernel category $\mathbf{D}$, each endo homset $\mathcal{E} n d o(X)$, for $X \in \mathbf{D}$, is a Foulis semigroup. The mapping $X \mapsto \mathcal{E}$ ndo $(X)$ yields a presheaf $\mathbf{D} \rightarrow$ Sets.

The lack of functoriality in this construction is problematic. One possible way to address it is via another notion of morphism between Foulis semigroups, like Galois connections between orthomodular lattices in the category OMLatGal. We shall not go deeper into this issue. Also the possible sheaf-theoretic aspects involved in this situation (see also [17]) form a topic on its own that is not pursued here. We briefly consider some examples.

For the dagger kernel category Hilb of Hilbert spaces, the set $\mathcal{B}(H)$ of (bounded/continuous linear) endomaps on a Hilbert space $H$ forms a Foulis semigroup-but of course

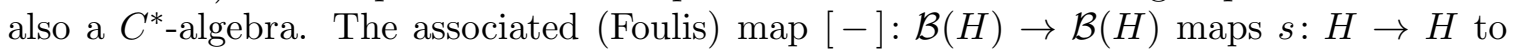
$[s]: H \rightarrow H$ given by $[s](x)=k\left(k^{\dagger}(x)\right)$, where $k$ is the kernel map $\{x \mid s(x)=0\} \hookrightarrow H$. 
For the category Rel of sets and relations the endomaps on a set $X$ are the relations $R \subseteq X \times X$ on $X$. The associated $[R] \subseteq X \times X$ is $\left\{(x, x) \mid \neg \exists_{y} . R(x, y)\right\}$.

An interesting situation arises when we apply the previous proposition to the dagger kernel category OMLatGal of orthomodular lattices (with Galois connections between them). One gets that for each orthomodular lattice $X$ the endo-homset $\mathcal{E}$ do $(X)=$ $\operatorname{OMLatGal}(X, X)$ forms a Foulis semigroup. This construction is more than 40 years old, see [13] or e.g. [4, Chapter II, Section 19] or [27, Chapter 5, §§18], where it is described in terms of Galois connections. In the present setting it comes for free, from the structure of the category OMLatGal. Hence we present it as a corollary, in particular of Proposition 4.2 and Theorem 3.5 .

Corollary 4.3. For each orthomodular lattice $X$ the set of (Galois) endomaps $\mathcal{E}$ ndo $(X)=$ $\operatorname{OMLatGal}(X, X)$ is a Foulis semigroup with composition as monoid, dagger $(-)^{\dagger}$ as involution, and self-adjoint idempotent $[s]: X \rightarrow X$, for $s: X \rightarrow X$, defined as in 4.1]. Equivalently, $[s]$ can be described via the Sasaki hook $\supset$ or and-then operator \&:

$$
[s]_{*}(x)=[s]^{*}(x)=s^{*}(1) \supset x^{\perp}=s^{*}(1)^{\perp} \vee\left(s^{*}(1) \wedge x^{\perp}\right)=\left(x \& s^{*}(1)\right)^{\perp} .
$$

Proof We recall from (4.1) that the operation [-] on endomaps $s: X \rightarrow X$ is defined as $[s]=\operatorname{ker}(s) \circ \operatorname{ker}(s)^{\dagger}: X \rightarrow X$. In OMLatGal one has $\operatorname{ker}(s)=s^{*}(1)$-see Proposition 3.9 so that:

$$
\begin{aligned}
{[s]_{*}(x) } & =\left(\operatorname{ker}(s) \circ \operatorname{ker}(s)^{\dagger}\right)_{*}(x) \\
& =\left(\operatorname{ker}(s)_{*}\left(\operatorname{ker}(s)^{*}(x)^{\perp}\right)\right. \\
& =s^{*}(1)_{*}\left(s^{*}(1) \wedge s^{*}(1)^{*}(x)^{\perp}\right) \\
& =\left(s^{*}(1) \wedge\left(s^{*}(1) \wedge x^{\perp}\right)^{\perp}\right)^{\perp} \quad \text { by Lemma 3.4 } \\
& =s^{*}(1)^{\perp} \vee\left(s^{*}(1) \wedge x^{\perp}\right) .
\end{aligned}
$$

4.1. From Foulis semigroups to dagger kernel categories. Each involutive monoid $(S, \cdot, 1, \dagger)$ forms a dagger category with one object, and morphisms given by elements of $S$. Requirement (4) in Definition 4.1 says that this category has "semi" kernels, given by [-]. Hence it is natural to apply the Karoubi envelope to obtain proper kernels. It turns out that this indeed yields a dagger kernel category.

For a Foulis semigroup as in Definition 4.1, we thus write $\mathcal{K}^{\dagger}(S)$ for the dagger Karoubi envelope applied to $S$ as one-object dagger category. Thus $\mathcal{K}^{\dagger}(S)$ has self-adjoint idempotents $s \in S$ as objects, and morphisms $f: s \rightarrow t$ given by elements $f \in S$ with $f \cdot s=f=t \cdot f$.

Theorem 4.4. This $\mathcal{K}^{\dagger}(S)$ is a dagger kernel category. The mapping $S \mapsto \mathcal{K}^{\dagger}(S)$ yields a functor $\mathbf{F s g} \rightarrow$ DKC.

Proof The zero element $0=[1] \in S$ is obviously a self-adjoint idempotent, and thus an object of $\mathcal{K}^{\dagger}(S)$. It is a zero object because for each $s \in \mathcal{K}^{\dagger}(S)$ there is precisely one map $f: s \rightarrow 0$, namely 0 , because $f=0 \cdot f=0$.

For an arbitrary map $f: s \rightarrow t$ in $\mathcal{K}^{\dagger}(S)$ we claim that there is a dagger kernel of the form:

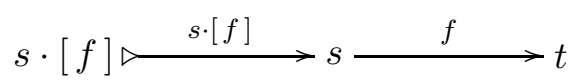

This will be checked in a number of small steps.

- $f \cdot(s \cdot[f])=(f \cdot s) \cdot[f]=f \cdot[f]=0$, by $\left(4^{\prime}\right)$ in Defintion 4.1 , 
- By the previous point there is an element $y \in S$ with $s \cdot[f]=[f] \cdot y$. Hence:

$$
[f] \cdot s \cdot[f]=[f] \cdot[f] \cdot y=[f] \cdot y=s \cdot[f]
$$

This is equation is very useful. It yields first of all that $s \cdot[f]$ is idempotent:

$$
(s \cdot[f]) \cdot(s \cdot[f])=s \cdot([f] \cdot s \cdot[f])=s \cdot s \cdot[f]=s \cdot[f] .
$$

This element is also self-adjoint:

$$
(s \cdot[f])^{\dagger}=([f] \cdot s \cdot[f])^{\dagger}=[f]^{\dagger} \cdot s^{\dagger} \cdot[f]^{\dagger}=[f] \cdot s \cdot[f]=s \cdot[f] .
$$

Hence $s \cdot[f] \in S$ is a self-adjoint idempotent, and thus an object of $\mathcal{K}^{\dagger}(S)$.

- $s \cdot[f]:(s \cdot[f]) \rightarrow s$ is also a dagger mono:

$$
\begin{aligned}
(s \cdot[f])^{\dagger} \cdot(s \cdot[f]) & =[f]^{\dagger} \cdot s^{\dagger} \cdot s \cdot[f] \\
& =[f] \cdot s \cdot s \cdot[f] \\
& =[f] \cdot s \cdot[f] \\
& =s \cdot[f] \\
& =\operatorname{id}_{s \cdot[f]} .
\end{aligned}
$$

- Finally, if $g: r \rightarrow s$ in $\mathcal{K}^{\dagger}(S)$ satisfies $f \circ g=f \cdot g=0$, then there is a $y \in S$ with $g=[f] \cdot y$. Then:

$$
s \cdot[f] \cdot g=s \cdot[f] \cdot[f] \cdot y=s \cdot[f] \cdot y=s \cdot g=g .
$$

Hence $g$ is the mediating map $r \rightarrow(s \cdot[f])$, since $(s \cdot[f]) \cdot g=g$. Uniqueness follows because $s \cdot[f]$ is a dagger mono.

As to functoriality, assume $h: S \rightarrow T$ is a morphism of Foulis semigroups. It yields a functor $H: \mathcal{K}^{\dagger}(S) \rightarrow \mathcal{K}^{\dagger}(T)$ by $s \mapsto h(s)$ and $f \mapsto h(f)$. This $H$ preserves all the dagger kernel structure because it preserves the Foulis semigroup structure.

By combining this result with Proposition 4.2 we have a way of producing new Foulis semigroups from old.

Corollary 4.5. Each self-adjoint idempotent $s \in S$ in a Foulis semigroup $S$ yields a Foulis semigroup of endo-maps:

$$
\mathcal{E} n d o(s)=\mathcal{K}^{\dagger}(S)(s, s)=\{t \in S \mid s \cdot t=t=t \cdot s\}
$$

with composition $\cdot$, unit $s$, involution $\dagger$ and $[t]_{s} \stackrel{\text { def }}{=} s \cdot[t] \cdot s$. The special case $s=1$ yields the original semigroup: $\mathcal{E}$ do $(1)=S$.

Proof We only check the formulation following (4.1):

$$
[t]_{s}=\operatorname{ker}(t) \circ \operatorname{ker}(t)^{\dagger}=s \cdot[t] \cdot(s \cdot[t])^{\dagger}=s \cdot[t] \cdot[t] \cdot s=s \cdot[t] \cdot s .
$$

The posets of kernel subobjects in a dagger kernel category are orthomodular lattices. This applies in particular to the category $\mathcal{K}^{\dagger}(S)$ and yields a way to construct orthomodular lattices out of Foulis semigroups. We first investigate this lattice structure in more detail, via (isomorphic) subsets of $S$.

Lemma 4.6. Let $S$ be a Foulis semigroup with self-adjoint idempotent $s \in S$, considered as object $s \in \mathcal{K}^{\dagger}(S)$. The subset

$$
K_{s} \stackrel{\text { def }}{=}\{s \cdot[t \cdot s] \mid t \in S\} \subseteq S
$$


is an orthomodular lattice with the following structure.

$$
\begin{array}{lrl}
\text { Order } & k_{1} \leq k_{2} & \Leftrightarrow k_{1}=k_{2} \cdot k_{1} \\
\text { Top } & 1_{s} & =s=s \cdot[s \cdot 0] \\
\text { Orthocomplement } & k^{\perp} & =s \cdot[k] \\
\text { Meet } & k_{1} \wedge k_{2} & =\left(k_{1} \cdot\left[\left[k_{2}\right] \cdot k_{1}\right]^{\perp \perp} .\right.
\end{array}
$$

In fact, $K_{s} \cong \operatorname{KSub}(s)$.

Proof It suffices to prove the last isomorphism $K_{s} \cong \mathrm{KSub}(s)$ and use it to translate the orthomodular structure from $\mathrm{KSub}(s)$ to $K_{s}$. Instead we proceed in a direct manner and show that each $K_{s}$ is an orthomodular lattice in a number of small consecutive steps, resembling the steps taken in [27, Chapter $5, \S \S 18]$. One observation that is used a number of times is:

$$
x \cdot y=0 \Longrightarrow y=[x] \cdot y
$$

for arbitrary $x, y \in S$, Indeed, if $x \cdot y=0$, then by requirement (4) in Definition 4.1 there is a $z$ with $y=[x] \cdot z$. But then $[x] \cdot y=[x] \cdot[x] \cdot z=[x] \cdot z=y$.

Let $s \in S$ now be a fixed self-adjoint idempotent.

(a) Each $k \in K_{s}$ is a self-adjoint idempotent, a dagger kernel $k: k \rightarrow s$, and also an idempotent $k: s \rightarrow s$ in $\mathcal{K}^{\dagger}(S)$.

Indeed, if $k=s \cdot[t \cdot s]$, then $(t \cdot s) \cdot k=t \cdot s \cdot[t \cdot s]=0$, so that $k=[t \cdot s] \cdot k$ by $(*)$. Hence:

$$
\begin{aligned}
k \cdot k & =s \cdot[t \cdot s] \cdot k=s \cdot k=k \\
k^{\dagger} & =([t \cdot s] \cdot k)^{\dagger}=([t \cdot s] \cdot s \cdot[t \cdot s])^{\dagger} \\
& =[t \cdot s]^{\dagger} \cdot s^{\dagger} \cdot[t \cdot s]^{\dagger}=[t \cdot s] \cdot s \cdot[t \cdot s]=k \\
k^{\dagger} \cdot k & =k \cdot k=k \\
k \cdot s & =k^{\dagger} \cdot s^{\dagger}=(s \cdot k)^{\dagger}=k^{\dagger}=k .
\end{aligned}
$$

Also, $k: k \rightarrow s$ is the kernel of $t \cdot s: s \rightarrow 1$, using the description of kernels in $\mathcal{K}^{\dagger}(S)$ from the proof of Theorem 4.4 ,

(b) The set $S$ carries a transitive order $t \leq r$ iff $r \cdot t=t$. This $\leq$ is a partial order on $K_{s}$.

Transitivity is obvious: if $t \leq r \leq q$, then $r \cdot t=t$ and $q \cdot r=r$ so that $q \cdot t=q \cdot r \cdot t=$ $r \cdot t=t$, showing that $t \leq q$.

Reflexivity $k \leq k$ holds for $k \in K_{s}$ since we have $k \cdot k=k$ as shown in (a). For symmetry assume $k \leq \ell$ and $\ell \leq k$ where $k, \ell \in K_{s}$. Then $\ell \cdot k=k$ and $k \cdot \ell=\ell$. Hence $k=k^{\dagger}=(\ell \cdot k)^{\dagger}=k^{\dagger} \cdot \ell^{\dagger}=k \cdot \ell=\ell$.

(c) For an arbitrary $t \in S$ put $t^{\perp} \stackrel{\text { def }}{=} s \cdot\left[t^{\dagger} \cdot s\right] \in K_{s}$. Hence from (a) we get equations $t^{\perp} \cdot t^{\perp}=t^{\perp}=\left(t^{\perp}\right)^{\dagger}$ and $s \cdot t^{\perp}=t^{\perp}=t^{\perp} \cdot s$ that are useful in calculations.

We will show $t \leq r \Rightarrow r^{\perp} \leq t^{\perp}$ and $k^{\perp \perp}=k$ for $k \in K_{s}$. 
Assume $t \leq r$, i.e. $t=r \cdot t$. Then, applying the equation $y=\left[\left[y^{\dagger} \cdot x^{\dagger}\right] \cdot x\right] \cdot y$ from requirement $\left(4^{\prime}\right)$ in Definition 4.1 for $y=\left[r^{\dagger} \cdot s\right]$ and $x=t^{\dagger} \cdot s$ yields:

$$
\begin{aligned}
{\left[r^{\dagger} \cdot s\right] } & =\left[\left[\left[r^{\dagger} \cdot s\right]^{\dagger} \cdot\left(t^{\dagger} \cdot s\right)^{\dagger}\right] \cdot t^{\dagger} \cdot s\right] \cdot\left[r^{\dagger} \cdot s\right] \\
& =\left[\left[\left[\left(t^{\dagger} \cdot s \cdot\left[r^{\dagger} \cdot s\right]\right)^{\dagger}\right]\right] \cdot t^{\dagger} \cdot s\right] \cdot\left[r^{\dagger} \cdot s\right] \\
& =\left[\left[\left((r \cdot t)^{\dagger} \cdot s \cdot\left[r^{\dagger} \cdot s\right]\right)^{\dagger}\right] \cdot t^{\dagger} \cdot s\right] \cdot\left[r^{\dagger} \cdot s\right] \\
& =\left[\left[\left(t^{\dagger} \cdot r^{\dagger} \cdot s \cdot\left[r^{\dagger} \cdot s\right]\right)^{\dagger}\right] \cdot t^{\dagger} \cdot s\right] \cdot\left[r^{\dagger} \cdot s\right] \\
& =\left[\left[\left(t^{\dagger} \cdot 0\right)^{\dagger}\right] \cdot t^{\dagger} \cdot s\right] \cdot\left[r^{\dagger} \cdot s\right] \quad \text { since } x \cdot[x]=0 \\
& =\left[[0] \cdot t^{\dagger} \cdot s\right] \cdot\left[r^{\dagger} \cdot s\right] \\
& =\left[1 \cdot t^{\dagger} \cdot s\right] \cdot\left[r^{\dagger} \cdot s\right] \\
& =\left[t^{\dagger} \cdot s\right] \cdot\left[r^{\dagger} \cdot s\right] .
\end{aligned}
$$

This gives us what we need to show $r^{\perp} \leq t^{\perp}$ :

$$
\begin{array}{rlrl}
t^{\perp} \cdot r^{\perp} & =t^{\perp} \cdot s \cdot\left[r^{\dagger} \cdot s\right] & \\
& =t^{\perp} \cdot\left[r^{\dagger} \cdot s\right] & & \text { since } t^{\perp} \in K_{s} \\
& =s \cdot\left[t^{\dagger} \cdot s\right] \cdot\left[r^{\dagger} \cdot s\right] & \\
& =s \cdot\left[r^{\dagger} \cdot s\right] & & \text { as we have just seen } \\
& =r^{\perp} . & &
\end{array}
$$

Next we notice that

$$
t^{\perp \perp}=s \cdot\left[\left(t^{\perp}\right)^{\dagger} \cdot s\right]=s \cdot\left[\left[t^{\dagger} \cdot s\right]^{\dagger} \cdot s^{\dagger} \cdot s\right]=s \cdot\left[\left[t^{\dagger} \cdot s\right] \cdot s\right] .
$$

Requirement $\left(4^{\prime}\right)$ in Definition 4.1, applied to $t$, says:

$$
s \cdot t=s \cdot\left[\left[t^{\dagger} \cdot s^{\dagger}\right] \cdot s\right] \cdot t=s \cdot\left[\left[t^{\dagger} \cdot s\right] \cdot s\right] \cdot t=t^{\perp \perp} \cdot t=t^{\perp \perp} \cdot s \cdot t .
$$

It says that $s \cdot t \leq t^{\perp \perp}$. In particular, this means $k \leq k^{\perp \perp}$ for $k \in K_{s}$. Since $(-)^{\perp}$ reverses the order we get:

$$
t^{\perp \perp \perp} \leq(s \cdot t)^{\perp}=s \cdot\left[(s \cdot t)^{\dagger} \cdot s\right]=s \cdot\left[t^{\dagger} \cdot s^{\dagger} \cdot s\right]=s \cdot\left[t^{\dagger} \cdot s\right]=t^{\perp} .
$$

If we finally apply this to $k \in K_{s}$, say for $k=s \cdot[t \cdot s]=\left(t^{\dagger}\right)^{\perp}$ we get:

$$
k^{\perp \perp}=\left(t^{\dagger}\right)^{\perp \perp \perp} \leq\left(t^{\dagger}\right)^{\perp}=k .
$$

(d) As motivation for the definition of meet, consider for $k_{1}, k_{2} \in K_{s}$ their meet as kernels:

$$
\begin{aligned}
r & \stackrel{\text { def }}{=} k_{1} \cdot k_{1}^{-1}\left(k_{2}\right) \\
& =k_{1} \cdot \operatorname{ker}\left(\operatorname{coker}\left(k_{2}\right) \cdot k_{1}\right) \quad \text { see pullback from Section } 2 \\
& =k_{1} \cdot \operatorname{ker}\left(\left(s \cdot\left[k_{2}^{\dagger}\right]\right)^{\dagger} \cdot k_{1}\right) \\
& =k_{1} \cdot k_{1} \cdot\left[\left[k_{2}\right] \cdot s \cdot k_{1}\right] \\
& =k_{1} \cdot\left[\left[k_{2}\right] \cdot k_{1}\right] .
\end{aligned}
$$

We force this $r$ into $K_{s}$ via double negation and hence define $k_{1} \wedge k_{2}=r^{\perp \perp}$. Showing that it is the meet of $k_{1}, k_{2}$ requires a bit of work.

- We have $k_{1} \cdot r=k_{1} \cdot k_{1} \cdot\left[\left[k_{2}\right] \cdot k_{1}\right]=k_{1} \cdot\left[\left[k_{2}\right] \cdot k_{1}\right]=r$, so that $r \leq k_{1}$ and thus also $k_{1} \wedge k_{2}=r^{\perp \perp} \leq k_{1}^{\perp \perp}=k_{1}$. 
- We first observe that

$$
\left[k_{2}\right] \cdot s \cdot s \cdot r=\left[k_{2}\right] \cdot s \cdot s \cdot k_{1} \cdot\left[\left[k_{2}\right] \cdot k_{1}\right]=\left[k_{2}\right] \cdot k_{1} \cdot\left[\left[k_{2}\right] \cdot k_{1}\right]=0 .
$$

Hence by applying $\dagger$ we get $\left(r^{\dagger} \cdot s\right) \cdot\left(s \cdot\left[k_{2}\right]\right)=0$. Via $(*)$ we obtain $s \cdot\left[k_{2}\right]=$ $\left[r^{\dagger} \cdot s\right] \cdot s \cdot\left[k_{2}\right]$, and thus also

$$
k_{2}^{\perp}=s \cdot k_{2}^{\perp}=s \cdot s \cdot\left[k_{2}\right]=s \cdot\left[r^{\dagger} \cdot s\right] \cdot s \cdot\left[k_{2}\right]=r^{\perp} \cdot k_{2}^{\perp} .
$$

This says $k_{2}^{\perp} \leq r^{\perp}$, from which we get $k_{1} \wedge k_{2}=r^{\perp \perp} \leq k_{2}^{\perp \perp}=k_{2}$.

- If also $\ell \in K_{s}$ satisfies $\ell \leq k_{1}$ and $\ell \leq k_{2}$, i.e. $k_{1} \cdot \ell=\ell=k_{2} \cdot \ell$, then, by Definition $4.1\left(4^{\prime}\right)$,

$$
\begin{aligned}
{\left[k_{2}\right] \cdot k_{1} \cdot \ell=\left[k_{2}\right] \cdot k_{2} \cdot \ell } & =\left(\left[k_{2}\right] \cdot k_{2}\right)^{\dagger \dagger} \cdot \ell \\
& =\left(k_{2}^{\dagger} \cdot\left[k_{2}\right]^{\dagger}\right)^{\dagger} \cdot \ell \\
& =\left(k_{2} \cdot\left[k_{2}\right]\right)^{\dagger} \cdot \ell \\
& =0^{\dagger} \cdot \ell \\
& =0 .
\end{aligned}
$$

Hence $\ell=\left[\left[k_{2}\right] \cdot k_{1}\right] \cdot \ell$ by $(*)$ and so $\ell=k_{1} \cdot \ell=s \cdot k_{1} \cdot \ell=s \cdot k_{1} \cdot\left[\left[k_{2}\right] \cdot k_{1}\right] \cdot \ell=s \cdot r \cdot \ell$. Thus $\ell \leq s \cdot r \leq r^{\perp \perp}=k_{1} \wedge k_{2}$.

(e) We get $k^{\perp} \wedge k=0$, for $k \in K_{s}$, as follows. Since $k \cdot s \cdot[k]=k \cdot[k]=0$ one has $k^{\perp}=s \cdot[k]=[k] \cdot s \cdot[k]=[k] \cdot k^{\perp}$ by $(*)$. Hence:

$$
k^{\perp} \wedge k=\left(k^{\perp} \cdot\left[[k] \cdot k^{\perp}\right]\right)^{\perp \perp}=\left(k^{\perp} \cdot\left[k^{\perp}\right]\right)^{\perp \perp}=0^{\perp \perp}=0 .
$$

(f) Finally, orthomodularity holds in $K_{s}$. We assume $k \leq \ell(i . e . k=\ell \cdot k)$ and $k^{\perp} \wedge \ell=0$, for $k, \ell \in K_{s}$, and have to show $\ell \leq k$ (i.e. $\ell=k \cdot \ell$, and thus $k=\ell$ ). To start, $k=k^{\dagger}=(\ell \cdot k)^{\dagger}=k^{\dagger} \cdot \ell^{\dagger}=k \cdot \ell$, so that $k \cdot \ell^{\perp}=k \cdot s \cdot[\ell]=k \cdot[\ell]=k \cdot \ell \cdot[\ell]=k \cdot 0=0$. Using $(*)$ yields $\ell^{\perp}=[k] \cdot \ell^{\perp}=[k] \cdot s \cdot[\ell]$, and also $\ell^{\perp}=\left(\ell^{\perp}\right)^{\dagger}=([k] \cdot s \cdot[\ell])^{\dagger}=$ $[\ell]^{\dagger} \cdot s^{\dagger} \cdot[k]^{\dagger}=[\ell] \cdot k^{\perp}$. Hence:

$$
\begin{aligned}
k^{\perp} \cdot \ell=k^{\perp} \cdot \ell^{\perp \perp}=k^{\perp} \cdot s \cdot\left[\ell^{\perp}\right] & =k^{\perp} \cdot\left[\ell^{\perp}\right] \\
& =k^{\perp} \cdot\left[[\ell] \cdot k^{\perp}\right] \\
& \leq\left(k^{\perp} \cdot\left[[\ell] \cdot k^{\perp}\right]\right)^{\perp \perp} \\
& =k^{\perp} \wedge \ell \\
& =0 .
\end{aligned}
$$

By $(*)$ we get $\ell=\left[k^{\perp}\right] \cdot \ell$ so that $\ell=s \cdot \ell=s \cdot\left[k^{\perp}\right] \cdot \ell=k^{\perp \perp} \cdot \ell=k \cdot \ell$, as required to get $\ell \leq k$.

Finally we need to show $K_{s} \cong \operatorname{KSub}(s)$. As we have seen in (a), each $k \in K_{s}$ yields (an equivalence class of) a kernel $k: k \rightarrow s$. Conversely, each kernel $\operatorname{ker}(f)=s \cdot[f]=s \cdot[f \cdot s]$ of a map $f: s \rightarrow t$ in $\mathcal{K}^{\dagger}(S)$ - see the proof of Theorem 4.4 is an element of $K_{s}$. This yields an order isomorphism: if $k_{1} \leq k_{2}$ for $k_{1}, k_{2} \in K_{s}$, then $k_{1}=k_{2} \cdot k_{1}$ so that we get a commuting triangle:

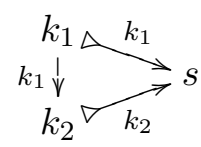


showing that $k_{1} \leq k_{2}$ in $\operatorname{KSub}(s)$. Conversely, if there is an $f: k_{1} \rightarrow k_{2}$ with $k_{2} \cdot f=k_{1}$, then $k_{2} \cdot k_{1}=k_{2} \cdot k_{2} \cdot f=k_{2} \cdot f=k_{1}$, showing that $k_{1} \leq k_{2}$ in $K_{s}$.

4.2. Generators. Recall that a generator in a category is an object $I$ such that for each pair of maps $f, g: X \rightarrow Y$, if $f \circ x=g \circ x$ for all $x: I \rightarrow X$, then $f=g$. Every singleton set is a generator in Sets, and also in Rel. The complex numbers $\mathbb{C}$ form a generator in the category Hilb of Hilbert spaces of $\mathbb{C}$. And the two-element orthomodular lattice is a generator in OMLatGal by Lemma 3.10 .

We shall write DKCg $\hookrightarrow$ DKC for the subcategory of dagger kernel categories with a given generator, and with morphisms preserving the generator, up to isomorphism.

Lemma 4.7. The dagger kernel category $\mathcal{K}^{\dagger}(S)$ associated with a Foulis semigroup has the unit $1 \in S$ as generator. The functor $\mathbf{F s g} \rightarrow$ DKC from Theorem 4.4 restricts to Fsg $\rightarrow$ DKCg.

Proof Assume $f, g: s \rightarrow t$ in $\mathcal{K}^{\dagger}(S)$ with $f \circ x=g \circ x$ for each map $x: 1 \rightarrow s$. Then, in particular for $x=s$ we get $f=f \circ s=g \circ s=g$. Every morphism $h: S \rightarrow T$ of Foulis semigroups satisfies $h(1)=1$, so that the induced functor $\mathcal{K}^{\dagger}(S) \rightarrow \mathcal{K}^{\dagger}(T)$ preserves the generator.

Lemma 4.8. The mapping $\mathbf{D} \mapsto \mathrm{KSub}(I)$ yields a functor $\mathbf{D C K g} \rightarrow$ OMLat.

Proof If $F: \mathbf{D} \rightarrow \mathbf{E}$ is a functor in $\mathbf{D C K g}$, then one obtains a mapping $\operatorname{KSub}_{\mathbf{D}}(I) \rightarrow$ $\operatorname{KSub}_{\mathbf{E}}(I)$ by:

$$
(M \bowtie \stackrel{m}{\longrightarrow} I) \longmapsto(F M \stackrel{F m}{\longrightarrow} F I \stackrel{\cong}{\longrightarrow} I) .
$$

Since all the orthomodular structure in kernel posets $\operatorname{KSub}(X)$ is defined in terms of kernels and daggers, it is preserved by $F$.

By composition we obtain the original ("old") way to construct an orthomodular lattice out of a Foulis semigroup, see [15].

Corollary 4.9. The composite functor $\mathbf{F s g} \rightarrow$ DCKg $\rightarrow$ OMLat maps a Foulis semigroup $S$ to the orthomodular lattice $[S]=\{[t] \mid t \in S\}=K_{1} \cong \mathrm{KSub}(1)$ from Lemma 4.6, over the generator 1 .

In the reverse direction we have seen in Corollary 4.3 that the set $\mathcal{E} n d o(X)$ of $(\mathrm{Ga}-$ lois) endomaps on an orthomodular lattice $X$ is a Foulis semigroup, but functoriality is problematic. However, we can now solve a problem that was left open in [20], namely the construction of a dagger kernel category out of an orthomodular lattice $X$. Theorem 4.4 says that the dagger Karoubi envolope $\mathcal{K}^{\dagger}(\mathcal{E} n d o(X))$ is a dagger kernel category. Its objects are self-adjoint idempotents $s: X \rightarrow X$, and its morphisms $f:(X, s) \rightarrow(X, t)$ are maps $f: X \rightarrow X$ in OMLatGal with $t \circ f=f=f \circ s$.

\section{Conclusions}

There is a relatively recent line of research applying categorical methods in quantum theory, see for instance [5, 1, 33, 9, 21, 6]. This paper fits into this line of work, with a focus on quantum logic (following [20]), and establishes a connection to early work on quantum structures. It constructs new (dagger kernel) categories of orthomodular lattices and of 
self-adjoint idempotents in Foulis semigroups (also known as Baer $*_{\text {-semigroups). These }}$ categorical constructions are shown to generalise translations between orthomodular lattics and Foulis semigroups from the 1960s. They provide a framework for the systematic study of quantum (logical) structures.

The current (categorical logic) framework may be used to address some related research issues. We mention three of them.

- As shown, the category OMLatGal of orthomodular lattices and Galois connections has (dagger) kernels and biproducts $\oplus$. An open question is whether it also has tensors $\otimes$, to be used for the construction of (logics of) compound systems, see [35]. The existence of such tensors is a subtle matter, given the restrictions described in [31.

- A dagger kernel category gives rise to not just one orthomodular lattice (or Foulis semigroup), but to a collection, indexed by the objects of the category, see for instance the presheaf description in Proposition 4.2. The precise, possibly sheaf-theoretic (see [17]), nature of this indexing is not fully understood yet.

- So-called effect algebras have been introduced as more recent generalisations of orthomodular lattices, see [11] for an overview. An open question is how such quantum structures relate to the present approach.

Acknowledgements. Many thanks to Chris Heunen for discussions and joint work [20], and to John Harding for spotting a mistake in an earlier version (see Remark 3.15).

\section{REFERENCES}

[1] S. Abramsky and B. Coecke. A categorical semantics of quantum protocols. In Logic in Computer Science, pages 415-425. IEEE, Computer Science Press, 2004.

[2] A. Baltag and S. Smets. LQP: the dynamic logic of quantum information. Math. Struct. in Comp. Sci., 16:491-525, 2006.

[3] G. Birkhoff and J. von Neumann. The logic of quantum mechanics. Ann. Math., 37:823-843, 1936.

[4] T.S. Blyth and M.F. Janowitz. Residuation Theory. Pergamum Press, 1972.

[5] J. Butterfield and C.J. Isham. A topos perspective on the Kochen-Specker theorem: I. quantum states as generalized valuations. Int. Journ. Theor. Physics, 37(11):2669-2733, 1998.

[6] B. Coecke, D. Pavlović, and J. Vicary. A new description of orthogonal bases. Math. Struct. in Comp. Sci., 2009, to appear. Available from http://arxiv.org/abs/0810.0812.

[7] B. Coecke and S. Smets. The Sasaki hook is not a [static] implicative connective but induces a backward [in time] dynamic one that assigns causes. Int. Journ. of Theor. Physics, 43(7/8):1705-1736, 2004.

[8] G.D. Crown. On some orthomodular posets of vector bundles. Journ. of Natural Sci. and Math., 15(12):11-25, 1975 .

[9] A. Döring and C.J. Isham. A topos foundation for theories of physics: I - IV. Journ. of Math. Physics, 49:053515-053518, 2008.

[10] A. Dvurečenskij. Gleason's Theorem and Its Applications. Number 60 in Mathematics and its Applications. Kluwer Acad. Publ., Dordrecht, 1992.

[11] A. Dvurečenskij and S. Pulmannová. New Trends in Quantum Structures. Kluwer Acad. Publ., Dordrecht, 2000.

[12] P. D. Finch. Quantum logic as an implication algebra. Bull. Amer. Math. Soc., 2:101-106, 1970.

[13] D. J. Foulis. Baer*-semigroups. Proc. Amer. Math. Soc., 11:648-654, 1960.

[14] D. J. Foulis. A note on orthomodular lattices. Portugaliae Mathematica, 21:65-72., 1962.

[15] D. J. Foulis. Relative inverses in Baer *-semigroups. Michigan Math. Journ., 10(1):65-84, 1963.

[16] P.J. Freyd. Abelian Categories: An Introduction to the Theory of Functors. Harper and Row, New York, 1964. Available via www.tac.mta.ca/tac/reprints/articles/3/tr3.pdf

[17] W.H. Graves and S.A. Selesnick. An extension of the Stone representation for orthomodular lattices. Coll. Mathematicum, XXVII:21-30, 1973. 
[18] S. Hayashi. Adjunction of semifunctors: categorical structures in nonextensional lambda calculus. Theor. Comp. Sci., 41:95-104, 1985.

[19] C. Heunen. Categorical Quantum Models and Logics. PhD thesis, Radboud Univ. Nijmegen, 2010.

[20] C. Heunen and B. Jacobs. Quantum logic in dagger kernel categories. Order, 2010, to appear. Available from http://arxiv.org/abs/0902.2355.

[21] C. Heunen, N.P. Landsman, and B. Spitters. A topos for algebraic quantum theory. Comm. in Math. Physics, 291:63-110, 2009. Available from http://arxiv.org/abs/0709.4364

[22] R. Hoofman. Non-Stable Models of Linear Logic. PhD thesis, Univ. Utrecht, 1992.

[23] R. Hoofman and I. Moerdijk. A remark on the theory of semi-functors. Math. Struct. in Comp. Sci., $5(1): 1-8,1995$.

[24] K. Husimi. Studies on the foundation of quantum mechanics I. Proc. physicomath. Soc. Japan, 19:766789, 1937.

[25] B. Jacobs. Semantics of the second order lambda calculus. Math. Struct. in Comp. Sci., 1(3):327-360, 1991.

[26] B. Jacobs. Categorical Logic and Type Theory. North Holland, Amsterdam, 1999.

[27] G. Kalmbach. Orthomodular Lattices. Academic Press, London, 1983.

[28] M. Karoubi. K-theory. An Introduction. Springer, 1978.

[29] J. Lambek and P.J. Scott. Introduction to higher order Categorical Logic. Number 7 in Cambridge Studies in Advanced Mathematics. Cambridge Univ. Press, 1986.

[30] S. Mac Lane and I. Moerdijk. Sheaves in Geometry and Logic. A First Introduction to Topos Theory. Springer, New York, 1992.

[31] C. Randall and D.J. Foulis. Tensor products of quantum logics do not exist. Notices Amer. Math. Soc., 26(6):A-557, 1979.

[32] D.S. Scott. Relating theories of the $\lambda$-calculus. In J.R. Hindley and J.P. Seldin, editors, To H.B. Curry: Essays on Combinatory Logic, Lambda Calculus and Formalism, pages 403-450, New York and London, 1980. Academic Press.

[33] P. Selinger. Dagger compact closed categories and completely positive maps (extended abstract). In P. Selinger, editor, Proceedings of the 3rd International Workshop on Quantum Programming Languages (QPL 2005), number 170 in Elect. Notes in Theor. Comp. Sci., pages 139-163. Elsevier, Amsterdam, 2007. DOI http://dx.doi.org/10.1016/j.entcs.2006.12.018

[34] P. Selinger. Idempotents in dagger categories (extended abstract). In P. Selinger, editor, Proceedings of the 4th International Workshop on Quantum Programming Languages (QPL 2006), number 210 in Elect. Notes in Theor. Comp. Sci., pages 107-122. Elsevier, Amsterdam, 2008. DOI http://dx.doi.org/10.1016/j.entcs.2008.04.021.

[35] Z. Shmuely. The tensor product of distributive lattices. Algebra Universalis, 9:281-296, 1979.

This work is licensed under the Creative Commons Attribution-NoDerivs License. To view a copy of this license, visit http://creativecommons.org/licenses/by-nd/2.0/ or send a letter to Creative Commons, 171 Second St, Suite 300, San Francisco, CA 94105, USA, or Eisenacher Strasse 2, 10777 Berlin, Germany 